\title{
Heat exchange mediated by a quantum system
}

\author{
George Y. Panasyuk f George A. Levin, and Kirk L. Yerkes \\ Aerospace Systems Directorate, Air Force Research Laboratory, Wright-Patterson Air Force Base, OH 45433
}

(Dated: April 28, 2022)

\begin{abstract}
We consider heat transfer between two thermal reservoirs mediated by a quantum system using the generalized quantum Langevin equation. The thermal reservoirs are treated as ensembles of oscillators within the framework of the Drude-Ullersma model. General expressions for the heat current and thermal conductance are obtained for arbitrary coupling strength between the reservoirs and the mediator and for different temperature regimes. As an application of these results we discuss the origin of Fourier's law in a chain of large, but finite subsystems coupled to each other by the quantum mediators. We also address a question of anomalously large heat current between the STM tip and substrate found in a recent experiment. The question of minimum thermal conductivity is revisited in the framework of scaling theory as a potential application of the developed approach.

PACS numbers: 05.70.Ln, 05.10.Gg, 65.80.-g
\end{abstract}

\section{INTRODUCTION}

The development of non-equilibrium thermodynamics mainly goes along two distinct directions. One direction is the study of the energy transfer through microscopic systems, such as nanotubes, molecules, or quantum dots [1, 2]. Beyond a purely academic interest in the problem, research suggests that nanoscale and molecular systems are good candidates for many technological advances, such as molecular wires, molecular diodes, rectifiers, and switches [3, 4]. The other direction, with longer history, deals with thermalization of, and energy flow through finite, macroscopic subsystems. Examples of such an approach are the Caldeira-Legett [5] and Nieuwenhuizen-Allahverdian [6, 7] models in which a thermal reservoir or macroscopic system is described as a large or infinite ensemble of harmonic modes.

In microscopic systems, such as chains of multilevel systems [8, 9], harmonic oscillators [10-12], or spins [13] the relaxation processes of individual elements and the processes of mutual equilibration between different elements are inseparable from each other and all take place on a microscopic time scale. In contrast, the local equilibrium requirement for macroscopic systems implies that the equilibration processes proceed on two vastly different time scales. The local equilibrium is established on the microscopic time scale, while the equilibration between the macroscopic subsystems takes a much longer time.

One of the most visible problems of non-equilibrium thermodynamics is the microscopic derivation of Fourier's law, specifying that the heat flux $\mathbf{j}$ through both fluids and solids is given by $\mathbf{j}=-\kappa \nabla T(\mathbf{r})$, where the temperature $T$ varies smoothly on microscopic scale and $\kappa$ is the thermal conductivity. Despite the ubiquitous occurrence of this phenomenon, very few rigorous mathematical derivations of this law are known [14]. While for 3D generic models Fourier's law is expected to be true, this law may not be valid for 1D and 2D

\footnotetext{
*Electronic address: George.Panasyuk.ctr@wpafb.af.mil
}

systems [1].

A recently developed approach to study heat transport at the microscopic level is based on either the classical or the quantum Langevin equation. The quantum Langevin equation was first considered in Ref. [15] for a weakly damped harmonic oscillator. Later [16], it was used to formulate transport, collective motion, and the Brownian motion from a unified, statistical-mechanical point of view. In Refs. [5-7, 1719], the Langevin equation was used for studying the thermalization of a particle coupled harmonically to a thermal reservoir and other closely-related problems. This approach was generalized in Refs. [11, 20-22] in order to explore the nonequilibrium steady-state heat current and temperature profiles in chains of harmonic oscillators placed between two thermal baths. Recently, a new method for an exact solution to the Lindblad and Redfield master equations for open quadratic system of $n$ fermions in terms of diagonalization of a $4 n \times 4 n$ matrix has been developed [23, 24]. The method has been applied to Heisenberg $X Y$ spin 1/2 chain coupled to heat baths at its ends. Generally, this approach can be considered as an alternative to the quantum Langevin equation.

In this paper we investigate the non-equilibrium steadystate heat transfer between two thermal reservoirs described as ensembles of harmonic modes mediated by a quantum system, which is also considered in the harmonic approximation. This is a Hamiltonian system with fixed total energy, but increasing entropy. Our approach is based on the quantum Langevin equation and uses the Drude-Ullersma model (DUM) for the bath mode spectrum. This is a generalization for the nonequilibrium situation of the approach employed in Ref. [7] to the study of statistical thermodynamics of a quantum Brownian particle coupled to a single thermal reservoir.

The solution obtained within this model allows us to determine the heat conductance between two thermal baths at arbitrary strength of the coupling constant. The results presented here are valid for an arbitrary temperature difference and arbitrary cut-off frequency, which plays the role of the Debye frequency. As is found, temperature dependence of the conductance may possess a plateau at intermediate temperature range, similar to the "classical" plateau at high temperatures. Dependence of the thermal conductance on the coupling 
strength displays a maximum. We also show that the quantum thermal bath approach, in which a many-body problem is replaced by the one-body approximation where the effect of thermal baths is quantified by random forces, yields the same results for conductance as the rigorous (many-body) solution in the limit of large Debye frequency. On a more general note, this approximation can be successfully used for solving more complex problems without necessarily resorting on the rigorous solution that is based on the full-fledged Hamiltonian.

The solution to the problem of heat transfer between two thermal reservoirs with a quantum particle as the mediator is applied to a chain system consisting of macroscopic subsystems coupled to each other by quantum particles (mediators). The microscopic time scale $\tau$ describes the time it takes for the heat current facilitated by the mediators to come to a steadystate. Each subsystem has arbitrary large heat capacity and the equilibration time between them is much longer than $\tau$. In this case, Fourier's law follows naturally as the differential form of the energy conservation law.

We use these results to explain a recent experiment in which the heat flow in vacuum between an STM tip and a substrate was found to be about ten orders of magnitude greater than that expected from the blackbody radiation theory [25]. Our suggestion is that the heat flow in this experiment was mediated by a carbon monoxide molecule placed in the gap between the tip and substrate. In addition, we briefly discuss the problem of minimum thermal conductivity attained when the coherence length of the phonons is minimal and of the order of the interatomic distance. Finally, we also briefly mention a possible application of the developed model to study the Josephson junction, which provides an important example of strong coupling between the quantum system and thermal baths.

The paper is organized as follows. The model is introduced in Sec. II, where the generalized Langevin equation is derived and solved using the DUM. In Sec. III, expressions for the heat current between the thermal baths and heat conductance are derived and analyzed for different temperature regimes and different coupling strengths. In Sec. IV, we compare the solution obtained in the quantum thermal bath approach with the the rigorous solution. In Section V the specifics of strong coupling is described. Section VI is devoted to Fourier's law in a chain of macroscopic subsystems. Section VII discusses the application of our model to anomalously large heat flow between the STM tip and substrate. Sections VIII and IX discuss possible applications of the model to deal with the problem of minimum thermal conductivity and the Josephson junction, respectively.

\section{LANGEVIN EQUATION}

The total Hamiltonian of the system under consideration is similar to that in Refs. [11, 26]

$$
\mathcal{H}_{\text {tot }}=\mathcal{H}+\mathcal{H}_{\mathrm{B} 1}+\mathcal{H}_{\mathrm{B} 2}+\mathcal{V}_{1}+\mathcal{V}_{2}
$$

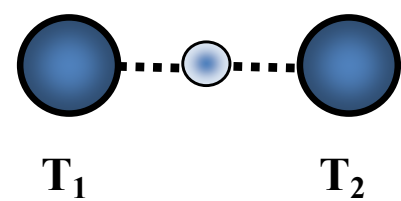

FIG. 1: (Color online) Diagram representation of the total Hamiltonian (1). The large circles correspond to the Hamiltonians of the thermal reservoirs, Eqs. (3), the small circle is the Hamiltonian (2) of the mediator, and the dotted lines correspond to the coupling Hamiltonians 4 .

Here

$$
\mathcal{H}=\frac{p^{2}}{2 m}+\frac{k x^{2}}{2}
$$

is the Hamiltonian of the quantum system (the mediator) described as a harmonic oscillator,

$$
\mathcal{H}_{\mathrm{B} \nu}=\sum_{i}\left[\frac{p_{\nu i}^{2}}{2 m_{\nu i}}+\frac{m_{\nu i} \omega_{\nu i}^{2} x_{\nu i}^{2}}{2}\right]
$$

are the Hamiltonians of the first $(\nu=1)$ and second $(\nu=2)$ baths, and

$$
\mathcal{V}_{\nu}=-x \sum_{i} C_{\nu i} x_{\nu i}+x^{2} \sum_{i} \frac{C_{\nu i}^{2}}{2 m_{\nu i} \omega_{\nu i}^{2}}
$$

describe interaction between the mediator and the baths. In Eq. (2), $x$ and $p$ are the coordinate and momentum operators and $m$ and $k$ are the mass and spring constant of the particle. In Eqs. (3) and (4), $x_{\nu i}$ and $p_{\nu i}$ are the coordinate and momentum operators, whereas $m_{\nu i}$ and $\omega_{\nu i}$ are the masses and frequencies of the oscillators for the $i$ th mode that belong to the $\nu$ th bath. Finally, $C_{\nu i}$ are the coupling coefficients that describe the interaction between the quantum system and the baths. The last contributions to the right-hand side of (4) are self-interaction terms, which guarantee that $\mathcal{H}_{\mathrm{B} \nu}+\mathcal{V}_{\nu}$ are positively defined operators. Fig. 11 contains graphical representation of the Hamiltonian (1). Here the large circles represent the Hamiltonians of the baths (3), the smaller central circle stands for the particle Hamiltonian (2), and the dashed lines describe the interaction between the central particle and the baths (4).

The Heisenberg equations for the coordinate and momentum operators corresponding to each bath and for our quantum system read

$$
\dot{x}_{\nu i}=\frac{p_{\nu i}}{m_{\nu i}}
$$

$$
\dot{p}_{\nu i}=-m_{\nu i} \omega_{\nu i}^{2} x_{\nu i}+C_{\nu i} x
$$

$$
\dot{x}=\frac{p}{m}
$$


and

$$
\dot{p}=-k x+\sum_{i \nu} C_{\nu i} x_{\nu i}-x \sum_{i \nu} \frac{C_{\nu i}^{2}}{m_{\nu i} \omega_{\nu i}^{2}} .
$$

Considering Eqs. (5) and (6) as a system of inhomogeneous equations with known inhomogeneity, its solution can be written as

$$
\begin{array}{r}
x_{\nu i}(t)=x_{\nu i}(0) \cos \left(\omega_{\nu i} t\right)+\frac{p_{\nu i}(0)}{m_{\nu i} \omega_{\nu i}} \sin \left(\omega_{\nu i} t\right)+ \\
\frac{C_{\nu i}}{m_{\nu i} \omega_{\nu i}} \int_{0}^{t} d s \sin \left[\omega_{\nu i}(t-s)\right] x(s)
\end{array}
$$

and

$$
\begin{gathered}
p_{\nu i}(t)=m_{\nu i} \dot{x}_{\nu i}(t)=-m_{\nu i} \omega_{\nu i} x_{\nu i}(0) \sin \left(\omega_{\nu i} t\right)+ \\
p_{\nu i}(0) \cos \left(\omega_{\nu i} t\right)+C_{\nu i} \int_{0}^{t} d s \cos \left[\omega_{\nu i}(t-s)\right] x(s) .
\end{gathered}
$$

Substituting (9) into (8) and integrating by parts, one obtains the quantum Langevin equation:

$$
m \ddot{x}=-k x(t)+\eta(t)-\int_{0}^{t} d s \gamma(t-s) \dot{x}(s)-\gamma(t) x(0),
$$

where

$$
\eta(t)=\sum_{i \nu} C_{\nu i}\left[x_{\nu i}(0) \cos \left(\omega_{\nu i} t\right)+\frac{p_{\nu i}(0)}{m_{\nu i} \omega_{\nu i}} \sin \left(\omega_{\nu i} t\right)\right]
$$

is the noise that comes from the baths and

$$
\gamma(t)=\sum_{i \nu} \frac{C_{\nu i}^{2}}{m_{\nu i} \omega_{\nu i}^{2}} \cos \left(\omega_{\nu i} t\right)
$$

is the friction kernel, which takes into account the interaction of the quantum particle with both thermal reservoirs.

\section{A. Drude-Ullersma model}

At this point we have made no specific assumptions about the properties of the Hamiltonians that describe the thermal reservoirs. The microscopic structure of the thermal reservoirs does not affect the nature of the energy exchange between them. Therefore, one can choose a specific, physically meaningful model of the Hamiltonians $\mathcal{H}_{\mathrm{B} 1}$ and $\mathcal{H}_{\mathrm{B} 2}$ without sacrificing the generality of the results.

Here we employ the Drude-Ullersma model (DUM) [7, 27, 28]. The model assumes that in the absence of the interaction with the quantum system, each bath consists of uniformly spaced modes and introduces the following $\omega$-dependence for the coupling coefficients:

$$
\omega_{\nu k}=k \Delta_{\nu}, \quad C_{\nu i}=\sqrt{\frac{2 \gamma_{\nu} m_{\nu i} \omega_{\nu i}^{2} \Delta_{\nu} D_{\nu}^{2}}{\pi\left(\omega_{\nu i}^{2}+D_{\nu}^{2}\right)}}
$$

where $k=1,2,3, \ldots N_{\nu}$. In Eq. (14), $\Delta_{\nu}$ are the mode spacing constants, $D_{\nu}$ are the characteristic cutoff frequencies qualitatively similar to the Debye frequency, and $\gamma_{\nu}$ are the coupling constants between a given reservoir and the mediator [7]. Hereafter we assume for simplicity that $D_{1}=$ $D_{2} \equiv D$. In the final results we take the limit $N_{\nu} \rightarrow \infty$ and $\Delta_{\nu} \rightarrow 0$.

Substituting Eq. (14) into Eq. (13) and replacing summation over $i$ by integration, one arrives at the following expression for $\gamma(t)$ :

$$
\gamma(t)=\gamma D e^{-D|t|},
$$

where $\gamma=\gamma_{1}+\gamma_{2}$. Using (14) and (15), Eq. (11) can be solved by Laplace transformation [29]:

$$
x(t)=\dot{g}(t) x(0)+\frac{1}{m} g(t) p(0)+\frac{1}{m} \int_{0}^{t} d s g(t-s) \eta(s) .
$$

Here

$$
g(t)=L^{-1}\left[\frac{1}{z^{2}+w_{0}^{2}+z \hat{\gamma}(z)}\right] \equiv L^{-1}[\tilde{g}(z)],
$$

where $\tilde{g}(z)$ is the Laplace transform $L$ of $g(t), L^{-1}$ is the inverse Laplace transform, and

$$
\hat{\gamma}(z)=\frac{1}{m} L[\gamma(t)]=\frac{D \hat{\gamma}}{D+z}, \hat{\gamma} \equiv \frac{\gamma}{m} .
$$

After substituting (18) into (17), $g(t)$ can be presented as

$$
g(t)=L^{-1}[\tilde{g}(z)]=\sum_{n=1}^{3} g_{n} e^{-\mu_{n} t}
$$

where

$$
\tilde{g}(z)=\frac{D+z}{(D+z)\left(z^{2}+\omega_{0}^{2}\right)+D \hat{\gamma} z} .
$$

Here $g_{n}$ are defined by the last two relations and $\mu_{n}$ are the roots of equation

$$
(\mu-D)\left(\mu^{2}+\omega_{0}^{2}\right)+\hat{\gamma} D \mu=0,
$$

where $\omega_{0}=\sqrt{k / m}$. Statistical thermodynamics of a quantum particle coupled to a thermal bath was considered in [7] in the limit of large $D$ when

$$
D>>\omega_{0}, 1 / \tau_{\mathrm{p}}=\gamma / m \equiv \hat{\gamma}, 1 / \tau_{\mathrm{x}} \equiv k / \gamma=\omega_{0}^{2} \tau_{\mathrm{p}} .
$$

In this case, (19) and 21) give

$$
\mu_{1,2} \approx \frac{1}{2 \tau_{\mathrm{p}}}(1 \mp r), \mu_{3} \approx D-1 / \tau_{\mathrm{p}}>>\left|\mu_{1,2}\right|
$$

and

$$
g_{1}=-g_{2} \approx \frac{\tau_{\mathrm{p}}}{r},
$$


where $r=\sqrt{1-4 \tau_{\mathrm{p}} / \tau_{\mathrm{x}}}$. If $\tau_{\mathrm{p}} / \tau_{\mathrm{x}}=\left(\omega_{0} \tau_{\mathrm{p}}\right)^{2} \leq 1 / 4, \mu_{1,2}$ are real and if $\tau_{p}<<\tau_{x}$, the quantities $\tau_{\mathrm{p}}$ and $\tau_{\mathrm{x}}$ can be interpreted as the characteristic relaxation times for the momentum and coordinate, respectively. Otherwise, when $\omega_{0} \tau_{\mathrm{p}}>1 / 2$, $\mu_{1,2}=1 / 2 \tau_{\mathrm{p}} \mp i / \tau_{0}$, where $\tau_{0}=\left(\omega_{0}^{2}-\hat{\gamma}^{2} / 4\right)^{-1 / 2}$ determine the oscillation time while $\tau_{p}$ again determines the damping time. In what follows, however, we consider a more general case when $D, \omega_{0}$, and $\tau_{\mathrm{p}}^{-1}$ may be comparable. In this case, the roots $\mu_{n}$ and coefficients $g_{n}$, where $n=1,2,3$, are determined as analytical solutions of (21) and from relation (19), respectively.

\section{HEAT CURRENT}

Using (5) and (6), one can easily show that the rate of change of the energy of a given thermal reservoir is given by

$$
\frac{d}{d t} \sum_{i}\left\langle\frac{p_{\nu i}^{2}}{2 m_{\nu i}}+\frac{m_{\nu i} \omega_{\nu i}^{2} x_{\nu i}^{2}}{2}\right\rangle \equiv-\left\langle\mathcal{P}_{\nu}\right\rangle,
$$

where

$$
\mathcal{P}_{\nu}=-\sum_{i} \frac{C_{\nu i}}{2 m_{\nu i}}\left\langle p_{\nu i} x+x p_{\nu i}\right\rangle
$$

is the work the quantum system performs on the $\nu$ th bath per unit of time (the power dispersed in the $\nu$ th bath) [20]. In the steady-state regime the power acquired by one reservoir is taken from the other, so that the steady-state heat current $J_{\text {th }}$ can be presented as $J_{\text {th }}=\left\langle\mathcal{P}_{1}\right\rangle=-\left\langle\mathcal{P}_{2}\right\rangle$ or in the symmetrical form

$$
J_{\mathrm{th}}=\frac{1}{2}\left\langle\mathcal{P}_{1}-\mathcal{P}_{2}\right\rangle
$$

Using solutions for $p_{\nu i}(t)$ and $x(t)$ from Eqs. (10) and (16), and omitting here the transient processes that wash out over the short time $\tau=\max \left(\tau_{\mathrm{p}}, \tau_{\mathrm{x}}\right)$ we get

$$
\begin{gathered}
\left\langle\mathcal{P}_{\nu}\right\rangle \approx-\frac{1}{2 m} \sum_{i=1} \frac{C_{\nu i}}{m_{\nu i}}\left[\cos \left(\omega_{\nu i} t\right) j_{\nu}^{(a)}\right. \\
\left.-m_{\nu i} \omega_{\nu i} \sin \left(\omega_{\nu i} t\right) j_{\nu}^{(b)}+C_{\nu i} j_{\nu}^{(c)}\right]
\end{gathered}
$$

where

$$
\begin{aligned}
& j_{\nu i}^{(a)}=\int_{0}^{t} d s g(t-s)\left\langle p_{\nu i}(0) \eta(s)+\eta(s) p_{\nu i}(0)\right\rangle, \\
& j_{\nu i}^{(b)}=\int_{0}^{t} d s g(t-s)\left\langle x_{\nu i}(0) \eta(s)+\eta(s) x_{\nu i}(0)\right\rangle,
\end{aligned}
$$

and

$$
\begin{aligned}
j_{\nu i}^{(c)}= & \left\langle\int_{0}^{t} d \tau \cos \omega_{\nu i}(t-\tau) x(\tau) \int_{0}^{t} d s g(t-s) \eta(s)+\right. \\
& \left.\int_{0}^{t} d s g(t-s) \eta(s) \int_{0}^{t} d \tau \cos \omega_{\nu i}(t-\tau) x(\tau)\right\rangle .
\end{aligned}
$$

The derivation of the steady-state expressions for $j_{1}^{(a)}, j_{1}^{(b)}$, $j_{1}^{(c)}$ and ultimately for $J_{\text {th }}$ depends on how the contact between the baths is established. A physically meaningful model should yield the same result regardless of how the coupling initially takes place. To verify this we have considered two options. In one case, the quantum system is attached simultaneously to both baths at time $t=0$. In the second case, the quantum particle is coupled first to the first bath, reaches thermal equilibrium with it, and at a later moment (which is again $t=0)$ it is coupled to the second bath.

We have established that the steady-state heat current is the same in either scenario. Below we give a derivation in the case of simultaneous coupling of the mediator to two thermal reservoirs at $t=0$. We can assume that for $t \leq 0$ the dynamic variables of the baths are determined by the usual expressions:

$$
x_{\nu i}(t)=\sqrt{\frac{\hbar}{2 m_{\nu i} \omega_{\nu i}}}\left(a_{\nu i}^{+} e^{i \omega_{\nu i} t}+a_{\nu i} e^{-i \omega_{\nu i} t}\right)
$$

and

$$
p_{\nu i}(t)=m_{\nu i} \dot{x}_{\nu i}(t) .
$$

Here the creation and annihilation operators $a_{\nu i}$ and $a_{\nu i}^{+}$satisfy $\left[a_{\nu i}, a_{\nu^{\prime} k}^{+}\right]=\delta_{i k} \delta_{\nu \nu^{\prime}}$. The operators' Gibbsian ensemble averages are determined by

$$
\left\langle a_{\nu i}^{+} a_{\nu^{\prime} k}+a_{\nu^{\prime} k} a_{\nu i}^{+}\right\rangle=\delta_{i k} \delta_{\nu \nu^{\prime}} \operatorname{coth}\left(\frac{\beta_{\nu} \hbar \omega_{\nu i}}{2}\right),
$$

where $\beta_{\nu}=\left(k_{\mathrm{B}} T_{\nu}\right)^{-1}$, which also result in

$$
\begin{array}{r}
\left\langle p_{\nu i}(0) p_{\nu^{\prime} j}(0)\right\rangle=m_{\nu i}^{2} \omega_{\nu i}^{2}\left\langle x_{\nu i}(0) x_{\nu^{\prime} j}(0)\right\rangle= \\
\frac{\hbar m_{\nu i} \omega_{\nu i}}{2} \delta_{i j} \delta_{\nu \nu^{\prime}} \operatorname{coth}\left(\frac{\beta_{\nu} \hbar \omega_{\nu i}}{2}\right)
\end{array}
$$

and

$$
\left\langle p_{\nu i}(0) x_{\nu^{\prime} j}(0)+x_{\nu^{\prime} j}(0) p_{\nu i}(0)\right\rangle=0 .
$$

Using these equations, as well as Eq. (12), the ensemble averages $\left\langle p_{\nu i}(0) \eta(t)+\eta(t) p_{\nu i}(0)\right\rangle,\left\langle x_{\nu i}(0) \eta(t)+\eta(t) x_{\nu i}(0)\right\rangle$ can be found and $\left\langle\mathcal{P}_{\nu}\right\rangle$ can be written as

$$
\left\langle\mathcal{P}_{\nu}\right\rangle=\left\langle\mathcal{P}_{\nu}\right\rangle^{(1)}+\left\langle\mathcal{P}_{\nu}\right\rangle^{(2)}
$$

where

$$
\begin{aligned}
\left\langle\mathcal{P}_{\nu}\right\rangle^{(1)}= & -\frac{\hbar}{2 m} \sum_{i=1} \frac{C_{\nu i}^{2}}{m_{\nu i}} \operatorname{coth}\left(\frac{\beta_{\nu} \hbar \omega_{\nu i}}{2}\right) \times \\
& {\left[\cos \left(\omega_{\nu i} t\right) \int_{0}^{t} d s g(t-s) \sin \left(\omega_{\nu i} s\right)-\right.} \\
& \left.\sin \left(\omega_{\nu i} t \int_{0}^{t} d s g(t-s) \cos \left(\omega_{\nu i} s\right)\right)\right]
\end{aligned}
$$

and

$$
\left\langle\mathcal{P}_{\nu}\right\rangle^{(2)}=-\frac{\hbar}{2 m} \sum_{i=1} \frac{C_{\nu i}^{2}}{m_{\nu i}} j_{\nu i}^{(c)}
$$


Evaluating the integrals in (38) using (19) and omitting exponentially decaying contributions, one can find that all time-varying terms, such as those proportional to $\sin \left(\omega_{\nu i} t\right) \cos \left(\omega_{\nu i} t\right)$, etc. cancel each other out. It means that the steady-state heat current is truly time-independent and does not contain any fluctuating contributions. Using Eq. 14 and substituting integration for summation we obtain

$$
\left\langle\mathcal{P}_{\nu}\right\rangle^{(1)}=-\frac{\hbar \gamma_{\nu} D^{2}}{\pi m} \sum_{n=1}^{3} g_{n} \mu_{n}^{2} \int_{0}^{\infty} \frac{d \omega \omega \operatorname{coth}\left(\beta_{\nu} \hbar \omega / 2\right)}{\left(\omega^{2}+D^{2}\right)\left(\omega^{2}+\mu_{n}^{2}\right)}
$$

Similarly, Eq. 39 can be rewritten as follows

$$
\begin{aligned}
\left\langle\mathcal{P}_{\nu}\right\rangle^{(2)}=- & \frac{\hbar \gamma_{\nu} D^{2}}{2 m}\left\langle\int_{0}^{t} d \tau S(t-\tau) x(\tau) \int_{0}^{t} d s g(t-s) \eta(s)\right. \\
& \left.+\int_{0}^{t} d s g(t-s) \eta(s) \int_{0}^{t} d \tau \cos S(t-\tau) x(\tau)\right\rangle
\end{aligned}
$$

where

$$
S(t)=\sum_{i=1} \frac{C_{\nu i}^{2}}{\gamma_{\nu} D^{2} m_{\nu i}} \cos \left(\omega_{\nu i} t\right)=2 \delta(t)-D e^{-D t} .
$$

Using (12), 35), and (36), $\left\langle\mathcal{P}_{\nu}\right\rangle^{(2)}$ can be obtained in a similar way, reaching its steady-state value when $t \rightarrow \infty$, and the heat current is given by

$$
J_{\mathrm{th}}=-\frac{\hbar D^{2}}{2 \pi \tau_{\mathrm{p}}} \sum_{n=1}^{3} g_{n} \mu_{n}^{2} \int_{0}^{\infty} \frac{d \omega \omega\left[n_{1}(\omega)-n_{2}(\omega)\right]}{\left(D^{2}+\omega^{2}\right)\left(\mu_{n}^{2}+\omega^{2}\right)}
$$

where $n_{\nu}(\omega)=1 /\left[\exp \left(\hbar \omega \beta_{\nu}\right)-1\right]$ are the phonon occupation number for the respective thermal reservoir. Here we assume for simplicity that $\gamma_{1}=\gamma_{2}$. In a more general case when $\gamma_{1} \neq \gamma_{2}, J_{\text {th }}$ will be determined by the same expression (42) by substituting $1 / 2 \tau_{\mathrm{p}} \rightarrow 2 \gamma_{1} \gamma_{2} /(\gamma m)$. It can be shown explicitly that the same result for $\left\langle\mathcal{P}_{\nu}\right\rangle^{(2)}$ and, eventually, for $J_{\text {th }}$ can be obtained if one performs $\tau$-integration in $j_{\nu i}^{(c)}$ first and, finally, calculates the $i$-sum in 39). This provides an additional verification of the formula (41) and expression (42). If $\left|T_{1}-T_{2}\right| \ll\left(T_{1}+T_{2}\right) / 2 \equiv T$, Eq. (42) determines the heat conductance $K$ :

$$
\begin{array}{r}
K=-\lim _{\Delta T \rightarrow 0} \frac{J_{\mathrm{th}}}{\Delta T}=-\frac{\tau_{\mathrm{h}}^{2} k_{\mathrm{B}} D^{2}}{8 \pi \tau_{\mathrm{p}}} \times \\
\sum_{n=1}^{3} g_{n} \mu_{n}^{2} \int_{0}^{\infty} \frac{d \omega \omega^{2} \operatorname{cosech}^{2}(\beta \hbar \omega / 2)}{\left(D^{2}+\omega^{2}\right)\left(\mu_{n}^{2}+\omega^{2}\right)}
\end{array}
$$

where $\Delta T=T_{2}-T_{1}$ and $\tau_{\mathrm{h}}=\hbar / k_{\mathrm{B}} T$.

In the second scenario of consecutive coupling of the mediator to the thermal baths, one can consider initially a closed system that describes the equilibrium (Gibbsian) state of the first thermal bath plus the mediator. The corresponding set of eigenvalues and eigenmodes $\left\{\nu_{k}, e_{k}\right\}$, can be determined by diagonalization of the Hamiltonian $\mathcal{H}+\mathcal{H}_{\mathrm{B} 1}+\mathcal{V}_{1}$. As was found in Ref. [7], the frequencies $\omega_{1 k}$ of the unperturbed modes of the Hamiltonian $\mathcal{H}_{\mathrm{B} 1}$ are shifted due to the interaction with the mediator to the values

$$
\nu_{1 k}=\omega_{1 k}-\frac{\Delta_{1}}{\pi} \phi\left(\omega_{k}\right)
$$

where $\phi(\omega)$ is a certain known function of the parameters of the Hamiltonian. In the limit of small $\gamma_{1}, \phi(\omega) \sim \gamma_{1}$.

In the Appendix we have shown that after coupling of this thermalized combined system to the second thermal bath with a different temperature, the same steady-state heat current (42) will be established despite the small difference in the microscopic makeup of the two baths. This is important not only from the physical point of view that the steady-state energy current between two thermal reservoirs should not depend on the initial conditions, but also for the derivation of Fourier's law as shown below.

It should be noted that the existence of a unique steadystate independent of the initial conditions cannot be taken for granted. There is substantial literature devoted to this problem in classical and quantum systems [1, 30-34]. Ref. [34] considers the existence of the steady-state and thermal equilibration in a system that represents a quantum wire coupled to two baths. It appears that a necessary, but not sufficient condition of uniqueness of the steady-state is absence of the bound state in the spectrum of the thermal bath. In the model we are using here the bound state would manifest itself as an imaginary root in Eq. 21). This would result in non-decaying oscillatory contribution to the steady-state energy current and its dependence on initial conditions. However, the absence of the bound states does not solely guarantee the uniqueness of the steady-state or thermal equilibration [1, 33, 34]. In this regard, our results demonstrate explicitly the existence and uniqueness of the steady-state in the considered model.

\section{A. Different temperature regimes}

Expressions for the heat current and heat conductance can be simplified in the limits of high and low temperatures when $\hbar\left|\mu_{n}\right| / k_{\mathrm{B}} T_{\nu} \ll 1$ and $\hbar\left|\mu_{n}\right| / k_{\mathrm{B}} T_{\nu} \gg 1$, respectively. Here $\mu_{n}(n=1,2,3)$ are the roots of Eq. (21).

In the high temperature limit, Eqs. (42) and 43) reduce to

$$
J_{\mathrm{th}}=-K\left(T_{2}-T_{1}\right) ; \quad K \approx-\frac{D k_{\mathrm{B}}}{4 \tau_{\mathrm{p}}} \sum_{n=1}^{3} \frac{g_{n} \mu_{n}}{D+\mu_{n}} .
$$

The above sum can be written as

$$
\sum_{n=1}^{3} \frac{g_{n} \mu_{n}}{D+\mu_{n}}=-\left.L[\dot{g}(t)]\right|_{z=D}=-D \tilde{g}(D)
$$

when using the well known properties of the Laplace transform and also relation $\sum_{n=1}^{3} g_{n} \mu_{n}^{k}=\left[(-1)^{k}-1\right] / 2$, where $k=0,1,2$. Thus, $K$ in (45) can be written as

$$
K \approx \frac{k_{\mathrm{B}}}{4 \tau_{\mathrm{p}}} \frac{2 D^{2}}{\left[2\left(D^{2}+\omega_{0}^{2}\right)+\hat{\gamma} D\right]}
$$


In the limit 22) of large $D$ we obtain

$$
K \approx \frac{k_{\mathrm{B}}}{4 \tau_{\mathrm{p}}}=\frac{k_{\mathrm{B}} \hat{\gamma}}{4} .
$$

In the deep quantum regime (low temperatures), we find

$$
J_{\mathrm{th}} \approx \frac{\pi^{3} k_{\mathrm{B}}^{4}\left(T_{1}^{4}-T_{2}^{4}\right)}{30 \hbar^{3} \tau_{\mathrm{p}}} \sum_{n=1}^{3} \frac{g_{n}}{\mu_{n}^{2}} .
$$

Using again the Laplace transform and 201, we find

$$
\sum_{n=1}^{3} \frac{g_{n}}{\mu_{n}^{2}}=-\frac{d \tilde{g}(z=0)}{d z}=\frac{\hat{\gamma}}{\omega_{0}^{4}}
$$

and finally have, in the quantum regime,

$$
J_{\mathrm{th}} \approx \frac{\pi^{3} k_{\mathrm{B}}^{4}}{30 \hbar^{3} \omega_{0}^{4} \tau_{\mathrm{p}}^{2}}\left(T_{1}^{4}-T_{2}^{4}\right) \text { and } K \approx \frac{2 \pi^{3} k_{\mathrm{B}}^{4} T^{3}}{15 \hbar^{3} \omega_{0}^{4} \tau_{\mathrm{p}}^{2}} .
$$

The temperature dependence of $J_{\mathrm{th}}$ is the same as in the Stefan-Boltzmann law

$$
J_{\mathrm{SB}}=A \frac{\pi^{2} k_{\mathrm{B}}^{4}}{60 \hbar^{3} c^{2}}\left(T_{1}^{4}-T_{2}^{4}\right),
$$

where $A$ is the area of a black body from which radiation emits. We will use this observation in Sec. VII for discussion of experimental results found in [25].

\section{QUANTUM THERMAL BATHS}

Let us show now that the result for the heat current given by Eq. (42) in the limit $D \rightarrow \infty$ can be reproduced within the quantum thermal bath (QTB) approach. In the phenomenological QTB model the many-body problem described by the Hamiltonian (1) is replaced by a one body problem in which friction is introduced "by hand", instead of being a logical consequence of energy redistribution between the practically infinite number of modes. The other effect of a thermal bath is modeled by a random force. The equation of motion for the mediator takes form of the Langevin equation

$$
m \ddot{x}+\gamma \dot{x}+m \omega_{0}^{2} x=F_{1}(t)+F_{2}(t),
$$

where the stochastic forces (colored noise) $F_{1}$ and $F_{2}$ describe the effects of the two heat baths with temperatures $T_{1}$ and $T_{2}$, respectively. We take $\left\langle F_{\nu}(t)\right\rangle=0$ and $\left\langle F_{\nu}(t) F_{\nu^{\prime}}\left(t^{\prime}\right)\right\rangle=$ $\delta_{\nu \nu^{\prime}} K_{\nu}\left(t-t^{\prime}\right)$, where $\nu, \nu^{\prime}=1,2$ and $K_{\nu}(t)$ is determined by its Fourier transform as

$$
K_{\nu}(t)=\frac{1}{2 \pi} \int_{-\infty}^{\infty} d \omega \tilde{K}_{\nu}(\omega) e^{-i \omega t}
$$

with

$$
\tilde{K}_{\nu}(\omega)=\frac{\gamma}{2} \hbar \omega \operatorname{coth}\left(\hbar \omega / 2 k_{\mathrm{B}} T_{\nu}\right)
$$

The correlators of the Fourier transforms of the random forces are given by

$$
\left\langle\tilde{F}_{\nu}(\omega) \tilde{F}_{\nu^{\prime}}\left(\omega^{\prime}\right)\right\rangle=2 \pi \delta\left(\omega+\omega^{\prime}\right) \delta_{\nu \nu^{\prime}} \tilde{K}_{\nu}(\omega) .
$$

The solution of Eq. (53) is given by

$$
\tilde{x}(\omega)=-\frac{1}{m} \frac{\tilde{F}_{1}(\omega)+\tilde{F}_{2}(\omega)}{\omega^{2}+i \hat{\gamma} \omega-\omega_{0}^{2}}, \quad \hat{\gamma}=\gamma / m .
$$

The energy conservation law for the mediator is given by

$$
\frac{d}{d t}\left\langle\frac{m \dot{x}^{2}}{2}+\frac{m \omega_{0}^{2} x^{2}}{2}\right\rangle=-\gamma\left\langle\dot{x}^{2}\right\rangle+\left\langle F_{1} \dot{x}\right\rangle+\left\langle F_{2} \dot{x}\right\rangle,
$$

which in the steady-state corresponds to $\gamma\left\langle\dot{x}^{2}\right\rangle=\left\langle F_{1} \dot{x}\right\rangle+$ $\left\langle F_{2} \dot{x}\right\rangle$.

We define heat current $J_{\text {th }}^{(\mathrm{s})}$ as the energy transferred per unit of time from the first to the second bath: $J_{\text {th }}^{(\mathrm{s})}=$ $-d E_{1} / d t$, or

$$
J_{\mathrm{th}}^{(\mathrm{s})}=-\frac{1}{2} \gamma\left\langle\dot{x}^{2}\right\rangle+\left\langle F_{1} \dot{x}\right\rangle=\frac{1}{2}\left(\left\langle F_{1} \dot{x}\right\rangle-\left\langle F_{2} \dot{x}\right\rangle\right) .
$$

Here $E_{1}$ is the internal energy of the first bath and we take that the energy dissipated by the mediator is equally split between the two baths. In general, the energy dissipated by the mediator can be split between the thermal baths in arbitrary proportion, but then the correlators $(58)$ also will be proportional to the fraction of energy dissipated by the mediator in a given bath.

Using Eqs. (57) and (56), we obtain

$$
J_{\mathrm{th}}^{(\mathrm{s})}=\frac{i}{4 \pi m} \int_{-\infty}^{\infty} \frac{d \omega \omega\left[\tilde{K}_{1}(\omega)-\tilde{K}_{2}(\omega)\right]}{\omega^{2}+i \hat{\gamma} \omega-\omega_{0}^{2}} .
$$

Taking into account expression (55), the heat current (60) can be rewritten as

$$
J_{\mathrm{th}}^{(\mathrm{s})}=\frac{\hbar}{2 \pi \tau_{\mathrm{p}}^{2}} \int_{0}^{\infty} \frac{d \omega \omega^{3}\left[n_{1}(\omega)-n_{2}(\omega)\right]}{\left(\omega^{2}-\omega_{0}^{2}\right)^{2}+\hat{\gamma}^{2} \omega^{2}}
$$

and the corresponding conductance is

$$
K^{(\mathrm{s})}=\frac{\tau_{\mathrm{h}}^{2} k_{\mathrm{B}}}{8 \pi \tau_{\mathrm{p}}^{2}} \int_{0}^{\infty} \frac{d \omega \omega^{4} \operatorname{cosech}^{2}(\beta \hbar \omega / 2)}{\left(\omega^{2}-\omega_{0}^{2}\right)^{2}+\hat{\gamma}^{2} \omega^{2}} .
$$

Thus, the heat current given by Eq. 61) and obtained from the phenomenological quantum thermal bath approach coincides in the limit $D \rightarrow \infty$ with that given by Eq. [42), which is the result of rigorous solution of a microscopic many-body model of the thermal reservoirs. This is a solid indication that the quantum thermal baths approach can be used to address more complicated problems of energy transfer via quantum mediators without resorting to a full-blown treatment based on a many-body Hamiltonian such as the one given by Eq. (11). It should be noted that the QTB model has been recently 
successfully used by Dammak et al. [35] for sampling quantum fluctuations within the framework of molecular dynamics (MD) simulations. Using the QTB model, the authors reproduced several experimental data at low temperatures in a regime where quantum statistical effects cannot be neglected. Our result here suggests that the MD approach can account for quantum statistical effects in non-equilibrium situations as well.

Also we should mention that a powerful method for solving problems involving open quadratic systems for fermions has been developed by Prosen [23] and Prosen and Z̆uncovič [24]. It would be interesting to see how a problem of nonequilibrium bosonic systems, like the one considered here, can be reformulated in terms of this novel approach of "third quantization".

\section{WEAK AND STRONG COUPLING REGIMES}

In this section we return to the analysis of our results emphasizing the effects of the weak and strong coupling on heat transfer as well as some interesting features in the behavior of the heat conductance. The main purpose of this section is to clarify the conditions which may allow us to assign a certain temperature to the mediator. This is possible when the mediator is in a state of weak non-equilibrium. For a simple system with two degrees of freedom the weak non-equilibrium means that the virial theorem is approximately satisfied. This condition depends on the coupling strength $\gamma$ and temperature. Let us consider the average potential and kinetic energy:

$$
k_{\mathrm{B}} T_{\mathrm{x}}=\left\langle k x^{2}\right\rangle \text { and } k_{\mathrm{B}} T_{\mathrm{p}}=\left\langle p^{2} / m\right\rangle
$$

The imbalance between them has been proven to be useful in determining the statistics of a quantum particle coupled to a single heat bath [7]. Even when the total system - (heat bath + mediator) - is in thermal equilibrium, the virial theorem is not satisfied for the mediator, namely $T_{\mathrm{p}}(T)-T_{\mathrm{x}}(T)$ is non-zero for any finite $\gamma$. This is a manifestation of quantum entanglement between a particle and a single thermal bath [7]. The difference between the average kinetic and potential energy of the mediator can serve as a criterion for the coupling strength also in the non-equilibrium case, as shown below.

Eqs. (12), 16), and (34), and the relation $p=m \dot{x}$, one can obtain

$$
T_{\mathrm{x}}=\frac{\hbar \omega_{0}^{2} \tau_{\mathrm{p}} S}{\pi k_{\mathrm{B}}}, \tau_{\mathrm{p}} S=\sum_{n=1}^{3} g_{n}\left[I_{n}\left(1-\hat{\mu}_{n}^{2}\right)+I_{n 1}+I_{n 2}\right]
$$

and

$$
T_{\mathrm{p}}=-\frac{\hbar}{\pi k_{\mathrm{B}}} \sum_{n=1}^{3} g_{n} \mu_{n}^{2}\left[I_{n}\left(1-\hat{\mu}_{n}^{2}\right)+I_{n 1}+I_{n 2}\right]
$$

Here $\hat{\mu}_{n}=\mu_{n} / D$,

$$
\begin{array}{r}
I_{n}=\int_{0}^{\infty} \frac{x d x}{\left(x^{2}+1\right)\left(x^{2}+\hat{\mu}_{n}^{2}\right)}= \\
\frac{i[\arctan (\mathrm{a})-(\pi / 2) \operatorname{sign}(\mathrm{b})]-\ln \left(\left|\hat{\mu}_{\mathrm{n}}\right|^{2}\right)}{2\left(1-\hat{\mu}_{n}^{2}\right)}
\end{array}
$$

with $a=\left(\mu_{n \mathrm{r}}^{2}-\mu_{n \mathrm{i}}^{2}\right) /\left(\mu_{n \mathrm{r}} \mu_{n \mathrm{i}}\right), b=\mu_{n \mathrm{r}} \mu_{n \mathrm{i}}, \mu_{n \mathrm{r}}=\operatorname{Re}\left(\mu_{\mathrm{n}}\right)$, and $\mu_{n \mathrm{i}}=\operatorname{Im}\left(\mu_{\mathrm{n}}\right)$. Finally,

$$
I_{n \nu}=\int_{0}^{\infty} \frac{x d x}{\left(e^{x}-1\right)\left[x^{2}+\left(\tau_{h \nu} \mu_{n}\right)^{2}\right]}
$$

where $\tau_{\mathrm{h} 1,2}=\hbar / k_{\mathrm{p}} T_{1,2}$.

The weak and strong coupling can be defined in terms of the effective bath-particle interaction strength $\hat{\gamma}_{\mathrm{D}}$, where

$$
\hat{\gamma}_{\mathrm{D}}=D^{2} \hat{\gamma}\left(D^{2}+\omega_{0}^{2}\right)^{-1}
$$

If $\hat{\gamma}_{\mathrm{D}} \ll \omega_{0}$, one can find from 19 -21 that

$$
\mu_{1,2} \approx \mp i \omega_{0}+\hat{\gamma}_{\mathrm{D}} / 2, \mu_{3}=D-\hat{\gamma}_{\mathrm{D}}
$$

and

$$
g_{1,2} \approx \mp \frac{i}{2 \omega_{0}}-\frac{\hat{\gamma}_{\mathrm{D}}}{2\left(D^{2}+\omega_{0}^{2}\right)}, g_{3}=\frac{\hat{\gamma}_{\mathrm{D}}}{D^{2}+\omega_{0}^{2}}
$$

If $\hat{\gamma}_{\mathrm{D}} \ll \omega_{0}$, the mediator can be described as an oscillator with relatively small effective friction. However, this condition by itself is not sufficient to guarantee the virial theorem. Only if temperatures of both thermal reservoirs are sufficiently high, i.e.

$$
k_{\mathrm{B}} T_{1,2} / \hbar \gg \hat{\gamma}_{\mathrm{D}}
$$

we get approximately equal steady-state values of $T_{\mathrm{x}, \mathrm{p}}$ :

$$
k_{\mathrm{B}} T_{\mathrm{x}} \approx k_{\mathrm{B}} T_{\mathrm{p}} \approx \frac{1}{2}\left[U\left(T_{1}\right)+U\left(T_{2}\right)\right]
$$

Here $U(T)=\hbar \omega_{0} / 2+\hbar \omega_{0}\left[\exp \left(\hbar \omega_{0} / k_{\mathrm{B}} T\right)-1\right]^{-1}$ is the average energy of a quantum oscillator in thermal equilibrium. The inequality (71) can be considered as the usual condition of applicability of the Gibbsian statistics, when interaction energy between subsystems of a large closed system is small with respect to the internal energies of the subsystems [36]. Thus, as long as the virial theorem is preserved, one can assign to the mediator a certain temperature $T$ on the basis of Eq. (72): $U(T)=(1 / 2)\left[U\left(T_{1}\right)+U\left(T_{2}\right)\right]$. In the high temperature limit $k_{\mathrm{B}} T_{1,2} \gg \hbar \omega_{0}$, this leads to

$$
T_{\mathrm{x}} \approx T_{\mathrm{p}} \approx T \approx\left(T_{1}+T_{2}\right) / 2,
$$

as is expected.

For the case of moderate or strong coupling (overdamped mediator), $T_{\mathrm{x}, \mathrm{p}}$ acquire $\gamma$-dependence and the condition (72) is not satisfied any more. Fig. 2 shows the monotonic dependence of the relative energy imbalance $\Delta \hat{T}_{\mathrm{px}} \equiv\left(T_{\mathrm{p}}-\right.$ $\left.T_{\mathrm{x}}\right) /\left(T_{\mathrm{p}}+T_{\mathrm{x}}\right)$ on the coupling strength. The cut-off parameter 


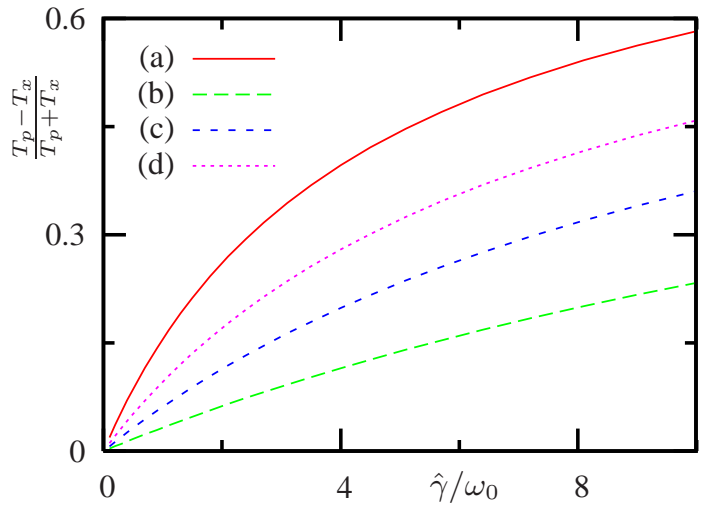

FIG. 2: (Color online) Dependence of $\Delta \hat{T}_{\mathrm{px}}$ on the coupling strength for different $D / \omega_{0}, \tau_{\mathrm{h} 1} \omega_{0}$, and $\tau_{\mathrm{h} 2} \omega_{0}$. (a) $D / \omega_{0}=10, \tau_{\mathrm{h} 1} \omega_{0}=$ $\tau_{\mathrm{h} 2} \omega_{0}=1$, (b) $D / \omega_{0}=1, \tau_{\mathrm{h} 1} \omega_{0}=\tau_{\mathrm{h} 2} \omega_{0}=1$ (c) $D / \omega_{0}=10$, $\tau_{h 1} \omega_{0}=\tau_{\mathrm{h} 2} \omega_{0}=0.5$, and (d) $D / \omega_{0}=1, \tau_{\mathrm{h} 1} \omega_{0}=1, \tau_{\mathrm{h} 2} \omega_{0}=$ 0.5 .

$D$ can loosely associate with the Debye frequency. The thermal bath modes with frequencies higher than $D$ are effectively decoupled from the mediator and do not play a significant role in the processes of thermalization and energy transfer. For this reason, when $D / \omega_{0}$ decreases, $\Delta \hat{T}_{\mathrm{px}}$ also decreases. This also follows from 68). Indeed, the coupling constant $\hat{\gamma} \equiv \gamma / m$ is renormalized by the factor $D^{2} /\left(D^{2}+\omega_{0}^{2}\right)$ and is effectively determined by $\hat{\gamma}_{\mathrm{D}}$ at a relatively small $\hat{\gamma}_{\mathrm{D}}$. As one also finds, $\Delta \hat{T}_{\mathrm{px}}$ decreases when $T_{1,2}$ grow in accordance to (71) - 73). Thus, $\Delta \hat{T}_{\mathrm{px}}$ can be considered as a measure of the coupling strength that takes into account all the relevant factors such as $D^{2} /\left(D^{2}+\omega_{0}^{2}\right)$ and values of $T_{1,2}$.

It is interesting to notice that the $\gamma$-dependence of the heat conductance (43) may possess a maximum. As we found, it appears at relatively small $\omega_{0}$ and relatively large $\hat{\gamma}$ (strong quantum entanglement between the particle and baths) when $\tau_{\mathrm{h}} \omega_{0} \lesssim 2, \hbar \hat{\gamma} / k_{\mathrm{B}} T \gtrsim 10$, and $\hat{\gamma} / D>1$ as is illustrated in Fig. 3 The maximum strength can be characterized by the quantity $\Delta \hat{K}=\left(K_{\max }-K_{\infty}\right) / K_{\max }$, where $K_{\max }$ and $K_{\infty}$ are the values of $K$ at its maximum and at $\hat{\gamma} / \omega_{0} \rightarrow \infty$, respectively. As our simulations show, $\Delta \hat{K}$ increases when $\tau_{\mathrm{h}} \omega_{0}$ decreases and the value $\left(\hat{\gamma} / \omega_{0}\right)_{\max }$ at which $K_{\max }$ is achieved shifts toward larger values when $D / \omega_{0}$ decreases. It is worth to mention that a similar maximum in the dependence of the heat current on the system-bath coupling strength was found in [24] for Heisenberg $X Y$ spin 1/2 chain.

Fig. 4 shows the temperature dependences for the normalized heat conductance at different $D$ and for $\omega_{0} \tau_{\mathrm{p}}=10$. The shown dependencies are based on the same expression (43) and its high- $D$ limit. As our analysis reveals, if $D / \omega_{0} \gtrsim 10$, $K(T)$ essentially coincides with the corresponding $D \rightarrow \infty$ limit (62). The straight line region corresponds to the low$T$ limit 51), which is the same for all curves. At large $T$, each curve reaches its classical plateau in accordance to [45]. An unusual feature, which is an additional plateau that appears in the intermediate range of $\tau_{\mathrm{p}} / \tau_{\mathrm{h}}$ and $D<<\omega_{0}$, is

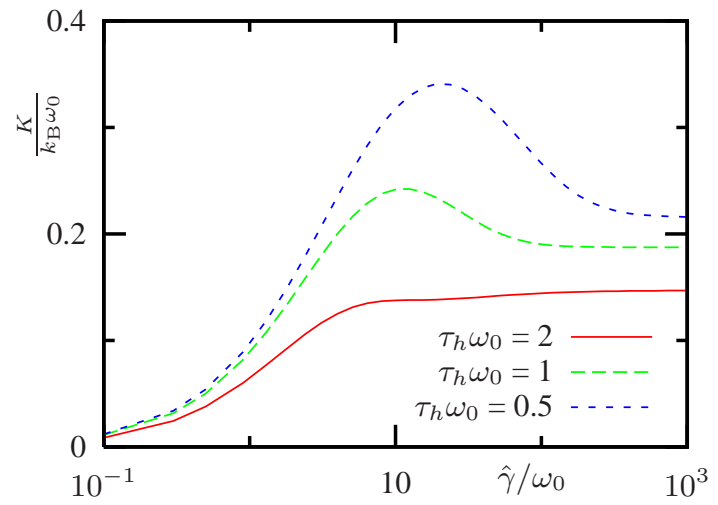

FIG. 3: (Color online) Dependence of the normalized heat conductance $K /\left(k_{\mathrm{B}} \omega_{0}\right)$ on the coupling strength at $D / \omega_{0}=1$ and different $\tau_{\mathrm{h}} \omega_{0}$.

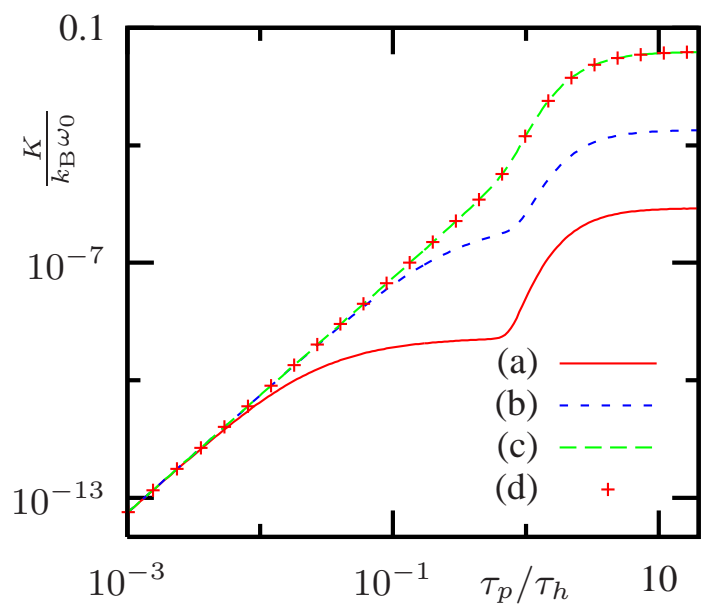

FIG. 4: (Color online) Temperature dependence for the normalized heat conductance $K /\left(k_{\mathrm{B}} \omega_{0}\right)$ at $\omega_{0} \tau_{\mathrm{p}}=10$ and different values of $D$. Note that $\tau_{\mathrm{p}} / \tau_{\mathrm{h}} \equiv k_{\mathrm{B}} T / \hbar \hat{\gamma}$. (a) $D \tau_{\mathrm{p}}=0.1$, (b) $D \tau_{\mathrm{p}}=1$, (c) $D \tau_{\mathrm{p}}=100$, and (d) $D \tau_{\mathrm{p}}=\infty$.

a generic result valid in a wide range of $\omega_{0} \tau_{\mathrm{p}}$. It can be explained in the following way. If $T$ is so large that $\omega_{0} \tau_{\mathrm{h}}<1$ or $\tau_{\mathrm{p}} / \tau_{\mathrm{h}}>\omega_{0} \tau_{\mathrm{p}}$, we have the "final" classical plateau in Fig. 4 described by (47). On the other hand, if $T$ is so small that $D \tau_{\mathrm{h}}>1$ or $\tau_{\mathrm{p}} / \tau_{\mathrm{h}}<D \tau_{p}$, we have the quantum regime (straight line in Fig. (4) described by (51). In the intermediate region, when $D \tau_{\mathrm{h}}<1$ but $\omega_{0} \tau_{\mathrm{h}}>1$ or $D \tau_{\mathrm{p}}<\tau_{\mathrm{p}} / \tau_{\mathrm{h}}<\omega_{0} \tau_{\mathrm{p}}$, one can approximate $\omega^{2}+\mu_{1,2}^{2} \approx \mu_{1,2}^{2}$ under the integrals in (43) for $n=1$ or 2 and

$$
K \approx-\frac{\tau_{\mathrm{h}}^{2} k_{\mathrm{B}} D^{2}}{8 \pi \tau_{\mathrm{p}}}\left[J_{1} \sum_{n=1,2} g_{n}+g_{3} D^{2} J_{2}\right],
$$

where

$$
J_{k}=\int_{0}^{\infty} \frac{d \omega \omega^{2}}{\sinh ^{2}(\beta \hbar \omega / 2)\left(D^{2}+\omega^{2}\right)^{k}} .
$$

Taking into account that $g_{1}+g_{2}=-g_{3}$, one can rewrite (74) 


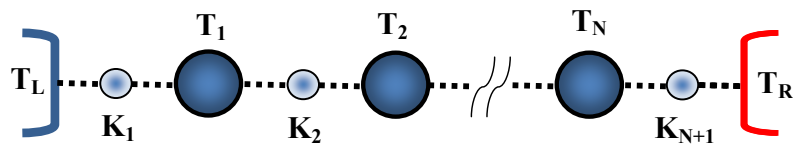

FIG. 5: (Color online) Chain of macroscopic subsystems ("nanoparticles") interconnected by mediators. Each subsystem indicated by a large circle, as well as both thermal reservoirs, correspond to Hamiltonian (3). Other symbols also have the same meaning as in Fig. 1 Temperatures $T_{\mathrm{L}}$ and $T_{\mathrm{R}}$ of the left and right thermal reservoirs are fixed because their heat capacity is considered infinite. The temperatures $T_{n}$ of the subsystems can slowly vary until the steady-state is established. Thermal conductances $K_{n}$ can vary from one connection to another.

as

$$
K \approx \frac{\tau_{\mathrm{h}}^{2} k_{\mathrm{B}} D^{2}}{8 \pi \tau_{\mathrm{p}}} g_{3} \int_{0}^{\infty} \frac{d \omega \omega^{4}}{\sinh ^{2}(\beta \hbar \omega / 2)\left(D^{2}+\omega^{2}\right)^{2}} .
$$

Finally, using that $D \tau_{\mathrm{h}}<1$ and approximating $\sinh (x) \approx x$, the integral in (76) can be estimated as $\pi /\left(D \tau_{\mathrm{h}}^{2}\right)$. Due to (70),

$$
K \approx \frac{k_{\mathrm{B}} D}{8 \pi \tau_{\mathrm{p}}} g_{3}=\frac{k_{\mathrm{B}} \hat{\gamma}}{4} \frac{D^{3} \hat{\gamma}}{2 \omega_{0}^{4}}
$$

does not, indeed, depend on temperature forming the plateau in Fig. 4 The physical origin for the small $K$ and intermediate plateau can be explained as follows. If $T$ increases above the Debye temperature $\theta_{\mathrm{D}}=D \hbar / k_{\mathrm{B}}$, i.e. $D \tau_{\mathrm{h}}=\theta_{\mathrm{D}} / T<$ 1 , all the baths modes begin to be excited. At the same time, if $T$ is still less than $\hbar \omega_{0} / k_{\mathrm{B}}$ (or $\tau_{\mathrm{h}} \omega_{0}>1$, which is always possible if $\left.\omega_{0} \gg D\right)$, the quantum system cannot be excited and, hence, it cannot absorb energy from either bath and transfer it to the other bath. This will lead to a small value of $K$ in (77). Moreover, because this situation stays unchanged when $T$ changes within the interval $\theta_{\mathrm{D}}<T<\hbar \omega_{0} / k_{\mathrm{B}}$ (or $\left.D \tau_{\mathrm{p}}<\tau_{\mathrm{p}} / \tau_{\mathrm{h}}<\omega_{0} \tau_{\mathrm{p}}\right), K$ must not depend on $T$ noticeably, which is in accordance to (77). Also, as one can notice, the effective bath-particle interaction strength $\hat{\gamma}_{D}$ is small if $D \gg \omega_{0}$ even if $\hat{\gamma}$ itself is not small. In this case, we have a very low decay rate for the $\mu_{1,2}$ modes and, correspondingly, very small heat current and heat conductance, again in accordance to (77). This result brings our situation close to the one with the bound states mentioned at the end of the first part of Sec. III. Indeed, when $\hat{\gamma}_{\mathrm{D}}=0$, we would have non-decaying oscillatory contributions to the steady-state, depending on the initial conditions, and zero heat current. However, even for a case with infinitely small $\hat{\gamma}_{\mathrm{D}}$, the unique but infinitely small steady-state current will be established after an infinitely large time interval.

\section{FOURIER'S LAW}

The results obtained in previous sections will be applied to an extended model that can shed additional light on the longstanding problem regarding the origin of Fourier's law [14].
The standard definition of a macroscopic body in the state of weak non-equilibrium [37] is that it can be divided into regions large enough to be considered macroscopic, but small enough to be described by a local temperature. In addition, these regions or subsystems must be weakly coupled to each other. The weak coupling in this context means that the characteristic time required for the subsystems to come to mutual equilibrium is much longer than the time of microscopic relaxation. Here we introduce a model that fills this conceptual framework with a microscopic content.

Fig. 5 illustrates our model. It consists of $N$ macroscopic subsystems and two thermal reservoirs (TR) coupled by the mediators. Each subsystem and each TR is described by the Hamiltonian given by Eq. (3) within the framework of the Drude-Ullersma model, Eq. (14). Each mediator is described by the Hamiltonian (2) and each coupling is described by the Hamiltonian (4) and Eq. (14). The total system, including the TRs, is Hamiltonian with constant total energy.

Each subsystem and each TR is initially prepared in the state of thermal equilibrium and is characterized by a temperature $T_{n}$ or $T_{\mathrm{L}}$ and $T_{\mathrm{R}}$, respectively. It means that the respective correlators have the form of Eq. (35).

The energy of a given subsystem, which consists of very large or infinite number of modes with frequencies $\omega_{k}=k \Delta$ with $k=1,2,3 \ldots$, is

$$
U(T) \sim \frac{\left(k_{\mathrm{B}} T\right)^{2}}{\hbar \Delta} .
$$

Here, the divergent zero-point energy term was dropped. Correspondingly, the heat capacity of a given subsystem

$$
C(T)=\frac{d U}{d T} \sim k_{\mathrm{B}} \frac{k_{\mathrm{B}} T}{\hbar \Delta} .
$$

Thus, the thermal reservoirs will be characterized by $\Delta \rightarrow 0$ and, correspondingly, an infinite heat capacity, while the subsystems need to be described by an arbitrarily small, but still finite $\Delta$. The energy difference between two subsystems (as in Fig. 10 with temperatures $T_{1}$ and $T_{2}$ is

$$
\Delta U \sim \frac{\left|\left(k_{\mathrm{B}} T_{1}\right)^{2}-\left(k_{\mathrm{B}} T_{2}\right)^{2}\right|}{\hbar \Delta} \sim \frac{k_{\mathrm{B}}^{2}\left|T_{1}-T_{2}\right| T_{\mathrm{av}}}{\hbar \Delta} .
$$

On the other hand, the heat current between the two subsystems can be estimated on the basis of Eqs. (45) and (48) as

$$
J_{\mathrm{th}} \sim \frac{k_{\mathrm{B}}}{\tau_{\mathrm{p}}}\left|T_{1}-T_{2}\right| .
$$

Thus, the characteristic time of mutual equilibration

$$
t_{\mathrm{eq}} \sim \frac{\Delta U}{J_{\mathrm{th}}} \sim \tau_{\mathrm{p}} \frac{k_{\mathrm{B}} T_{\mathrm{av}}}{\hbar \Delta}=\frac{\tau_{\mathrm{p}}}{\tau_{\mathrm{h}}} \Delta^{-1} \sim \Delta^{-1} .
$$

For the subsystems, we assume that the "Heisenberg" time scale $1 / \Delta$ is much larger than any other characteristic times, such as $\tau_{\mathrm{p}}, \tau_{\mathrm{x}}$, or $\tau_{\mathrm{h}}[6,7]$. Thus, the system described by the Hamiltonian represented by the diagram in Fig. 5 meets the conditions described in the first paragraph of this section. 
One of the main results that we have obtained by solving the Hamiltonian (1) is that the energy flows from higher to lower temperature TR. Since all the modes of a given TR are in thermal equilibrium at the same temperature, the thermodynamic relationship between energy and entropy $d U_{i}=T d S_{i}$ is satisfied for each of them and the total entropy in the steady-state increases

$$
\frac{d S}{d t}=\left|J_{\mathrm{th}}\right|\left|\frac{1}{T_{1}}-\frac{1}{T_{2}}\right|>0 .
$$

One can easily show that for the system represented in Fig. 5 the total entropy also increases even when the energy currents between the subsystems are all different. In the steady-state, which corresponds to all energy currents between two neighboring subsystems being equal, the entropy increases as

$$
\frac{d S}{d t}=\left|J_{\mathrm{th}}\right|\left|\frac{1}{T_{\mathrm{L}}}-\frac{1}{T_{\mathrm{R}}}\right|>0 .
$$

These results are valid as long as all modes remain in thermal equilibrium or close to equilibrium. The coupling constants given by Eq. 14 are proportional to the infinitesimal parameter $\Delta$,

$$
C_{\nu, i} \sim \Delta^{1 / 2}
$$

The effect of such coupling on the mediator is finite because all modes contribute constructively. The rate of change of the correlators (35) is much slower because each of the modes is coupled only to the mediator with vanishingly small coupling constant. One can see from Eqs. (6) - 12 that the rate of change of the correlators 35

$$
\frac{\partial}{\partial t}\left\langle p_{\nu i}(t) p_{\nu^{\prime} j}(t)\right\rangle \sim \frac{\partial}{\partial t}\left\langle x_{\nu i}(t) x_{\nu^{\prime} j}(t)\right\rangle \sim \Delta .
$$

Thus, if we consider the evolution of the system on the time scale $t$, such that

$$
\tau_{\mathrm{p}}<t \ll t_{\mathrm{eq}},
$$

which is long enough for the microscopic relaxation to take place, but short on the macroscopic time scale, all modes will remain approximately in thermal equilibrium determined by the initial conditions.

The same argument allows us to extend the solution of the Hamiltonian (1) shown as a diagram in Fig. 1 to the Hamiltonian that corresponds to Fig. 5 Imagine that after we prepared both TRs and all the subsystems in the state of thermal equilibrium at the corresponding temperatures we start turning on the couplings to mediators one-by-one from left to right. The energy flow between two subsystems indexed as $n$ and $n+1$ will be the same as for the Hamiltonian (1) because the effect of the subsystem $n$ being already coupled to the subsystem $n-1$ adds to the energy current a contribution of the order of $\Delta$ and therefore negligible on the time scale 79 . This means that the solutions of the Hamiltonian (1) can be directly applied to the Hamiltonian of the chain shown in Fig. 5 in the form of energy conservation condition

$$
\partial_{t} U_{n}=J_{n-1, n}-J_{n, n+1} .
$$

Here the energy currents $J_{n-1, n}$ are given by Eq. (42) and this equation is valid for arbitrary values of the initial temperatures. In order to obtain Fourier's law we have to take the temperature differences between the neighboring subsystems small and express the currents in terms of thermal conductances 43.

$$
\partial_{t} U_{n}=K_{n-1, n}\left(T_{n-1}-T_{n}\right)-K_{n, n+1}\left(T_{n}-T_{n+1}\right) .
$$

This equation can be rewritten in the differential form by introducing a continuous coordinate $x$, where $x=n d$ corresponds to the locations of the subsystems and $d$ is the distance between them. For identical mediators Eq. 81 can be recast as

$$
\begin{array}{r}
C(T) \partial_{t} T(x)=K(x-d / 2)[T(x-d)-T(x)]- \\
K(x+d / 2)[T(x)-T(x+d)],
\end{array}
$$

where $C(T)=d U / d T$ is the heat capacity of a subsystem. Then, Eq. 82 leads to the energy conservation condition with Fourier's form of the energy current

$$
\tilde{C}(T) \partial_{t} T(x)=\partial_{x}\left[\kappa(x) \partial_{x} T(x)\right]
$$

where $\tilde{C}=C / d$ is the specific heat of the chain, and the thermal conductivity $\kappa(T)=K(T) d$.

This analysis shows that as long as the energy flow between macroscopic subsystems satisfies the condition of entropy increase, namely, that the energy flows from higher to lower temperature, Fourier's law is a straightforward consequence of energy conservation. What has been proven in this paper is that the dynamics of the Hamiltonian system described by the Hamiltonian (1) and its extension shown as a diagram in Fig. 5 does indeed lead to the entropy increase. This statement is predicated on the condition of local thermal equilibrium or near equilibrium for the subsystems that exchange energy between themselves. We have stated in our treatment of the Hamiltonian (1) that this is an initial condition. As such, it remains true for the time interval indicated by inequality (79). However, the generally accepted understanding of the slow relaxation processes [37] in a macroscopic body implies that as each subsystem slowly gains or loses energy, it remains close to thermal equilibrium with a certain time-dependent temperature due to rapid thermalization on the microscopic time scale. Thus, the microscopic derivation of Fourier's law in the context of our model requires not only to prove that in a certain limit (smooth temperature variation) the energy current is proportional to the temperature gradient, which we have done. Equal, if not more important, task is to show that the subsystems described by the Drude-Ullersma model are capable of self-thermalization when coupled by the harmonic mediator. This proof will require the study of the dynamics of the Hamiltonian (1) on the time scale given by Eq. (78).

The model depicted in Fig. 5 resembles to some extent the models analyzed by Michel, Mahler and Gemmer [8], as well as those discussed by Dubi and Di Ventra [9]. The subsystems considered in [8, 9] were still microscopic with the heat capacity of the order of $k_{\mathrm{B}}$. Other publications [10-13] consider the 
energy transport in chains consisting of spins or harmonic oscillators. In all these cases the goal was to examine the energy transport on the "nano-scale". Our approach here is to examine a solvable Hamiltonian model of a non-equilibrium system that falls within the more traditional, "textbook" framework. The subsystems described by the Hamiltonian (3) can have arbitrarily large heat capacity determined by the infinitesimal parameter $\Delta$. Correspondingly, these subsystems remain in thermal equilibrium for the extended period of time, much greater than the microscopic relaxation time of the mediator and demonstrate that the energy flow leads to entropy increase and, hence, to Fourier's law, Eqs. 80 - 83. The mediators do not have to be in the state of thermal equilibrium for the Eq. (83) to be valid. As was discussed in Sec. V, in the strong coupling regime the mediator cannot be assigned a certain temperature. However, as long as the subsystems remain close to thermal equilibrium, Fourier's law is still valid.

The outstanding question of the quasistatic evolution and self-thermalization of such subsystems on the much longer time scale, Eq. (78), will be addressed elsewhere.

\section{A. Quantum thermal baths model}

In the limit of large $D$, Eq. 80 follows from the model shown in Fig. 6. The equation of motion for the $n$ 's mediator is practically the same as Eq. (53)

$$
m \ddot{x}_{n}+\gamma \dot{x}_{n}+m \omega_{0}^{2} x_{n}=F_{n-1}(t)+F_{n}(t),
$$

where the random force $F_{n}$ describes the effect of the corresponding subsystem $n$ on the mediator and the correlator of the force is determined by the temperature of the respective subsystem. Note, that the mediators are not directly coupled to each other by "springs", so that Eqs. (84) are not a system of coupled equations, but simply are $n$ identical equations, whose solution for the heat current $J_{n-1, n}$ is given by Eq. 61.

One can compare this model with the models of selfconsistent reservoirs [38-40]. Since our subsystems are macroscopically large, their effect on the mediator can be described in terms of a stochastic force. However, these forces are not arbitrarily introduced. We have shown that the exact solution of the Hamiltonian dynamics in the limit of large $D$ yields the same expression for the energy current as the quantum thermal baths model. In this sense the quantum thermal baths model can be a useful shortcut, but our model is defined by the Hamiltonian (11). It is also important to mention that the thermalization of these self-consistent reservoirs is considered a given in Refs. [38-40]. In fact, it has to be proven by analysis of the long term evolution of the Hamiltonian dynamics.

One should note that in our model the mediators are not coupled to each other directly. The energy flows only through the macroscopic subsystems. In this sense the chain of mediators operate, by design, in the minimum thermal conductivity limit because the neighboring mediators are always uncorrelated. If we were to associate the movement of the mediators

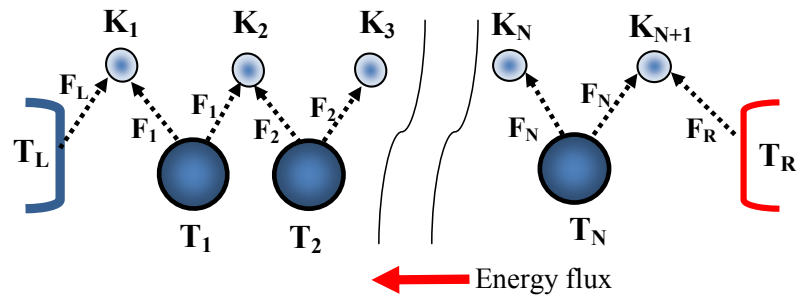

FIG. 6: (Color online) Diagram of the quantum thermal baths model equivalent to the Hamiltonian shown in Fig. 5 Here $F_{n}$ are the random forces that appear in Eq. 845 and the other elements are similar to ones from Fig. 5 Notice that the mediators are not coupled to each other directly.

with phonons, their mean free path would be minimum possible, equal to "interatomic" distance.

\section{HEAT CURRENT BETWEEN STM TIP AND SUBSTRATE}

The results obtained in previous sections can be used to clarify an interesting finding reported recently by Altfeder, Voevodin, and Roy [25]. In their experiment, the energy current in high vacuum between the Pt/Ir tip of the scanning tunneling microscope and gold film serving as a substrate was found to be anomalously large, exceeding by ten orders of magnitude the current given by the blackbody radiation theory. An interpretation of this phenomenon given in Ref. [25] involves "emission" of phonons by Au surface, facilitated by the electric field, and their "tunneling" through the vacuum gap to the tip. Here we would like to offer an alternative mechanism that seems to be capable to account quantitatively for the same effect.

An important feature of this experiment is that a carbon monoxide $(\mathrm{CO})$ molecule was always present between the $\mathrm{Pt}$ tip and the substrate. From the value of the tunneling conductance, the gap between the "last tip atom" and the surface of the Au film was inferred to be close to $3 \AA$ [41]. The diameter $a$ of a CO molecule is close to $3.7 \AA$ [42]. Thus, without the CO molecule, the distance between the tip and the substrate would be about $7 \AA$. There is also a strong electric field in the gap, which is due to the work function difference between $\mathrm{Pt}$ and $\mathrm{Au}(\approx 0.7 \mathrm{eV})$. The implication of this is that the $\mathrm{CO}$ molecule must be strongly coupled to both the tip and the Au surface. If we consider that this molecule serves as a mediator between the two thermal reservoirs (the tip and the substrate, similar to the arrangement in Fig. 1), the energy current between them can be estimated and compared with the experimental data. The coupling strength between the mediator and the thermal reservoirs determines how strongly damped the mediator is.

There are two vibration modes associated with CO molecule attached to the Pt tip [43]. One of them is the Pt$\mathrm{C}$ stretching vibration with frequency $480 \mathrm{~cm}^{-1}$. The other mode is the $\mathrm{C}-\mathrm{O}$ stretching motion with the frequency ap- 
proximately $2000 \mathrm{~cm}^{-1}$. Using the conversion coefficient 1 $\mathrm{K}=0.7 \mathrm{~cm}^{-1}$, we find that the lowest frequency $480 \mathrm{~cm}^{-1}$ corresponds to $685 \mathrm{~K}$ and the highest frequency $2000 \mathrm{~cm}^{-1}$ corresponds to $2.8 \times 10^{3} \mathrm{~K}$. In the experiment, the tip was maintained at room temperature, while the temperature of the substrate was substantially lower. Thus the higher mode was clearly not activated and we should take the frequency of the mediator in our model $\omega_{0} \approx 480 \mathrm{~cm}^{-1}$.

Since the main frequency $\hbar \omega_{0} / k_{\mathrm{B}} \approx 685 \mathrm{~K}$ is substantially greater than the temperature of the bulk of the tip $(\approx 300 \mathrm{~K})$ and the temperature of the substrate (in the range $90-210 \mathrm{~K}$ ), the first order approximation of the heat current mediated by the $\mathrm{CO}$ molecule can be estimated using Eq. 51,

$$
J_{\mathrm{CO}} \approx \frac{\pi^{3} k_{\mathrm{B}}^{4} \hat{\gamma}^{2}}{30 \hbar^{3} \omega_{0}^{4}}\left(T_{1}^{4}-T_{2}^{4}\right) .
$$

The expression for the energy current between the tip and the substrate due to phonon emission from the "hot" spot on the surface was used in Refs. [25, 44] in the form

$$
J_{\mathrm{A}} \approx \frac{\pi^{5} k_{\mathrm{B}}^{2}}{60 \hbar \theta_{\mathrm{D}}^{2}}\left(T_{1}^{4}-T_{2}^{4}\right) .
$$

Here $\theta_{\mathrm{D}}$ is the Debye temperature of the substrate. The underlying physics that leads to these two expressions is very different, but the temperature dependence is the same. The analysis based on Eq. (86) leads to a good description of the experimental results. Thus, the model of $\mathrm{CO}$-mediated heat transfer will also give the same results if the prefactors of Eqs. (85) and (86) are equal. This requires that

$$
\frac{\hat{\gamma}}{\omega_{0}}=\frac{\pi}{\sqrt{2}} \frac{\hbar \omega_{0}}{k_{\mathrm{B}} \theta_{\mathrm{D}}} .
$$

For gold $\theta_{\mathrm{D}} \approx 165 \mathrm{~K}$, and since $\hbar \omega_{0} / k_{\mathrm{B}} \approx 685 \mathrm{~K}$ we get

$$
\frac{\hat{\gamma}}{\omega_{0}} \approx 9 .
$$

Thus, other things being equal, the model based on COmediated heat exchange will give the same quantitative results as the model of phonon tunneling, provided that the coupling between the $\mathrm{CO}$ molecule and both the tip and substrate is strong enough to make the Pt-C vibrating mode overdamped according to Eq. (88). This is likely the case, considering (as mentioned above) that the size of the molecule is comparable to the gap between the tip and substrate and also taking into account the presence of the strong polarizing electric field.

The heat current given by Eq. (85) is $\sim 10^{10}$ times greater than that determined by the black-body radiation, Eq. (52), emitting from the area equal to the cross-section of the $\mathrm{CO}$ molecule $A=\pi a^{2} / 4$, with $a \approx 3.7 \AA$, provided that $\hat{\gamma} / \omega_{0} \sim$ 10.

Our conclusion is that the anomalously large heat current between the STM tip and the substrate can be understood just as well as the effect of mediation by the $\mathrm{CO}$ molecule. There are ways to modify the experiment in order to determine which of the two mechanisms is responsible for the effect. One is to carry out a similar measurement, but without the $\mathrm{CO}$ molecule lodged in the tunneling gap. Another option is to use the tip and substrate made of the same metal, so that there will be no work function potential difference $\Delta \Phi$ between them. The mechanism of phonon tunneling is based on the interaction between the electrically charged tip and its electrostatic image. The amount of charge is mainly determined by the large electric field $E=\Delta \Phi_{\mathrm{Au} / \mathrm{Pt}} / d$, where $d$ is the vacuum gap [25]. In the absence of such large field the mechanism of phonon tunneling should be greatly weakened.

\section{MINIMUM THERMAL CONDUCTIVITY}

The topic of minimum thermal conductivity can be first traced to Einstein's contribution (see Ref. [45] and references therein). In strongly disordered solids the phonon coherence length may become of the order of interatomic distance and, obviously, cannot be reduced any further. Correspondingly, the thermal conductivity reaches its minimum value, at least as far as its dependence on such length is concerned. A detailed treatment of this problem is given in Refs. [46, 47]. The same phenomenon leads to a minimal electric conductivity in disordered conductors - the so-called Mott-Ioffe-Regel limit [48, 49] - when the coherence length of the charge carriers become comparable to the interatomic distance.

Here we would like to add another perspective on this matter using the results obtained above and making use of the scaling approach previously developed for electron transport [50-52]. It should be emphasized that this section is not intended as a comprehensive treatment of this problem, but rather as a brief introduction to an alternative approach which may be useful in some systems.

Consider a microscopic block whose edges are along the principal axes $\{x, y, z\}$ of the thermal conductivity tensor. We can choose the sides of the block such that, on average, the random phase acquired by phonons due to an inelastic interactions along the way between the two opposite boundaries is the same, of the order of $2 \pi$, for all three pairs of the block boundaries. This choice for the sides of the block corresponds to the definition for the anisotropic phase coherence lengths $\ell_{\varphi, i}(i=\{x, y, z\})$, the distances over which phonons lose phase coherence.

Let $K_{\varphi, i}$ be the thermal conductance of such a phase coherent volume (PCV), so that the energy current through this block

$$
j_{\varphi, i}=K_{\varphi, i} \delta T_{i} .
$$

Here $\delta T_{i}$ is the temperature difference between the opposite edges of the PCV block. Notice that the notion of a temperature difference cannot be introduced for distances smaller than the phase coherence length. In order to express the macroscopic anisotropic thermal conductivity $\kappa_{i}$ in terms of the conductance of the PCV, consider a macroscopic block with sizes $\left\{L_{x}, L_{y}, L_{z}\right\}$ obtained by fitting together $N^{3}$ phase coherent volumes, so that $L_{x} / \ell_{\varphi, x}=L_{y} / \ell_{\varphi, y}=L_{z} / \ell_{\varphi, z}=N \gg 1$. 
By virtue of Fourier's law, the heat current in the $x$-direction through this macroscopic volume is

$$
J_{x}=\kappa_{x} \Delta T_{x} \frac{L_{y} L_{z}}{L_{x}} .
$$

Here we assume a linear temperature variation across the block. On the other hand, the heat currents through the individual PCVs combine linearly, so that

$$
J_{x}=K_{\varphi, x} \delta T_{x} N^{2}
$$

Taking into account that $\delta T_{x}=\Delta T_{x} / N$, we obtain

$$
\kappa_{x}=K_{\varphi, x} \frac{\ell_{\varphi, x}}{\ell_{\varphi, y} \ell_{\varphi, z}} .
$$

To put this result into a different perspective, the PCV is the minimal size block for which one can introduce the notion of thermal conductivity.

One can argue that the thermal conductance of the PCV of phonons in an anisotropic medium is isotropic, similar to the electric conductance in anisotropic metals [53], namely, $K_{\varphi, x}=K_{\varphi, y}=K_{\varphi, z}$. If this assertion were true, the anisotropy is defined by the following relationship:

$$
\frac{\kappa_{x}}{\kappa_{y}}=\frac{\ell_{\varphi, x}^{2}}{\ell_{\varphi, y}^{2}} ; \quad \frac{\kappa_{x}}{\kappa_{z}}=\frac{\ell_{\varphi, x}^{2}}{\ell_{\varphi, z}^{2}} .
$$

This relationship is in agreement with the quasiclassical results obtained from the kinetic equations $\kappa_{i} \sim c v_{i} \lambda_{i}$, where $c$ is the specific heat, $v_{i}$ is the anisotropic speed of sound, and $\lambda_{i}$ is the phonon mean free path. Considering that the mean free path is similar to the phase coherence length, so that $\lambda_{i} \sim \ell_{\varphi, i}=v_{i} \tau_{\varphi}$, we get $\kappa_{i} \sim c \ell_{\varphi, i}^{2} / \tau_{\varphi}$. Since both the relaxation (decoherence) time $\tau_{\varphi}$ and the specific heat are scalars, the relationship (93) follows.

Eq. (93) is most useful when applied to a system where at least one of the coherence lengths is temperature independent constant. It may be a highly disordered crystal in which the decoherence takes place over interatomic distances, or a layered structure in which the coherence length in one direction is fixed by the size of the layer. Further discussion of the consequences of Eq. (93) would be far outside the scope of this paper.

Now we will return to the case of highly disordered substances in which the coherence lengths in all directions are of the order of interatomic distances and do not change with temperature. There are numerous example of such substances where the minimum thermal conductivity is reached at temperatures above $30 \mathrm{~K}$ [45]. In vitreous, silica- and germaniabased glasses, the mean free path (or the phase coherence length) approaches the interatomic distance at $T \gtrsim 100 \mathrm{~K}$. For example, the phonon mean free path for amorphous selenium at $T \geq 50 \mathrm{~K}$ is temperature independent and equal to $5 \times 10^{-8} \mathrm{~cm}$, which corresponds approximately to the interatomic distance in this substance [54].

In the minimum thermal conductivity (MTC) regime the movements of the neighboring atoms are incoherent, so that there are no propagating phonons. Instead, every atom is acted upon by the non-equilibrium environment and the mechanism of this interaction can be described by the Hamiltonian given by Eq. (1) (see also Fig. 1). Thus, the conductance $K_{\varphi}$ of the PCV containing one atom can be well described by our model in which a single oscillator mediates the energy exchange between two thermal reservoirs. Then, by virtue of Eq. (92) the thermal conductivity is given by

$$
\kappa_{\min }=K_{\varphi} / \ell_{0}
$$

where $\ell_{0}$ is a constant of the order of the interatomic distance and $K_{\varphi}=K$ is given by the general expression, Eq. (43), and its limiting cases such as Eq. (48).

Let us consider the classical limit of high temperatures, Eq. (48), when Eq. (94) takes the form

$$
\kappa_{\min } \approx \frac{k_{\mathrm{B}}}{4 \tau_{\mathrm{p}} \ell_{0}}=\frac{k_{\mathrm{B}} \hat{\gamma}}{4 \ell_{0}} .
$$

This has to be compared with another expression for the MTC which is based on the atomic density $n$ and elastic constants 45, 47]

$$
\kappa_{\min }=0.4 k_{\mathrm{B}} n^{2 / 3}\left(v_{\mathrm{l}}+2 v_{\mathrm{t}}\right)
$$

Here $v_{\mathrm{l}}$ and $v_{\mathrm{t}}$ are the longitudinal and transverse speeds of sound, respectively. This is the sum of the contributions of the three spatial degrees of freedom. Taking into account that

$$
n^{2 / 3} \sim \frac{1}{\ell_{0}^{2}}
$$

$\kappa_{\text {min }}$ can be estimated as

$$
\kappa_{\min } \sim \frac{k_{\mathrm{B}}}{\ell_{0}} \frac{v_{\mathrm{av}}}{\ell_{0}} .
$$

Hereafter we will drop the numerical prefactors. The characteristic time scale $\ell_{0} / v_{\text {av }}$ is the time of flight of a phonon over the interatomic distance. The incoherence of the neighboring atoms means that the phonons lose their coherence over this time interval. This is exactly the meaning of the decoherence time $\tau_{\varphi} \sim \ell_{0} / v_{\mathrm{av}}$. In our model the relaxation time $\tau_{\mathrm{p}}$ is determined by the strength of the coupling between the oscillator and the thermal baths. It is the relaxation time of the momentum of the oscillator. Thus, in the MTC regime $\tau_{\varphi}$ and $\tau_{\mathrm{p}}$ are equivalent quantities,

$$
\tau_{\mathrm{p}} \sim \tau_{\varphi} \sim \frac{\ell_{0}}{v_{\mathrm{av}}}
$$

and the expressions for the MTC given by Eqs. (95) and 96 are qualitatively and even quantitatively similar.

Moreover, since the standard definition of the Debye frequency is $\omega_{\mathrm{D}} \equiv k_{\mathrm{B}} \theta_{\mathrm{D}} / \hbar \sim v_{\mathrm{av}} / \ell_{0}$, we see that in the MTC regime the relaxation time in our model must be

$$
\tau_{\mathrm{p}}^{-1}=\hat{\gamma} \sim \omega_{\mathrm{D}}
$$


The frequency $\omega_{0}$ is the highest frequency associated with atomic vibration and it must be also of the order of Debye frequency, $\omega_{0} \sim v_{\text {av }} / \ell_{0} \sim \omega_{\mathrm{D}}$. The last parameter of the model is the cut-off frequency $D$, which defines the maximum frequency of the modes of the thermal baths that are coupled to the mediator. In the context of solid substances this cut-off also must be of the order of the Debye frequency. Thus, we come to conclusion that the range of parameters within which the Hamiltonian model given by Eq. (1) is applicable to the description of the minimum conductivity regime is rather narrow and is given by

$$
\omega_{0} \sim \hat{\gamma} \sim D \sim \omega_{\mathrm{D}}
$$

As an example, we can take the data from Ref. [54] for selenium at temperatures above $100 \mathrm{~K}$. The value of thermal conductivity $\kappa_{\min } \sim 0.5 \times 10^{-2} \mathrm{WK}^{-1} \mathrm{~cm}^{-1}$. Comparing this value with Eq. (95), and taking into account that the characteristic interatomic distance $\ell_{0} \approx 5 \times 10^{-8} \mathrm{~cm}$, we find $\hat{\gamma} \sim 10^{13}$ $\mathrm{s}^{-1}$. The Debye temperature for selenium is $\theta_{\mathrm{D}} \sim 250 \mathrm{~K}$, so that $\omega_{\mathrm{D}} \sim 3 \times 10^{13} \mathrm{~s}^{-1}$ and the condition given by Eq. 99. is satisfied. Thus, in a highly disordered substance the oscillator enclosed inside the PCV is rather overdamped, $\omega_{0} \tau_{\mathrm{p}} \gtrsim 1$. Although this is only an estimate, we use it in order to illustrate the potential applications of our model. A more detailed comparison with the experimental data needs to involve the specific heat also calculated within the framework of the same model.

\section{JOSEPHSON JUNCTIONS}

Finally, we can mention that for some potential applications of our model, such as the Josephson junctions, all model's parameters are already experimentally known. This enables making valuable predictions about the physical behavior of the corresponding system. In the case of the Josephson junction, the particle's coordinate $x$ in the Langevin equation is substituted by $\phi$ which is the phase difference between the wave functions describing the state of condensate of Cooper pairs in the contacting superconductors kept at different temperatures. Here $\omega_{0}$ is the plasma frequency and $\hat{\gamma}=\gamma / m=$ $1 / R C$ with $R$ and $C$ are the junction resistance and capacitance, respectively [55]. Characteristic values for $\omega_{0}$ can vary between $10^{10} \mathrm{~s}^{-1}$ and $10^{14} \mathrm{~s}^{-1}$, depending on the current density. For tunnel junctions, when $\hat{\gamma} / \omega_{0}$ is usually in the range $0.001-0.1$, the damping is weak. For junctions with nontunneling conductivity and in the form of point contacts or thin-film microbridges [56], $\hat{\gamma} / \omega_{0}>>1$ and the damping is large.

\section{SUMMARY}

In conclusion, we have considered the heat transport between two thermal reservoirs mediated by a quantum particle using the generalized quantum Langevin equation. Both thermal reservoirs are described as ensembles of harmonic modes using the Drude-Ullersma model and the mediator is also treated in the harmonic approximation. The expressions obtained for the heat current and thermal conductance are valid for arbitrary coupling strength between the mediator and the reservoirs. The cutoff frequency, which characterizes the thermal reservoirs, can also be arbitrary. The obtained results are analyzed for different temperatures regimes and different strengths of the coupling parameter. The dependence of the thermal conductance on the coupling strength shows a maximum and the temperature dependence of this quantity reveals a plateau at intermediate temperatures, similar to the classical plateau that corresponds to the high-temperature limit.

The results are applied to a model of a chain made out of macroscopically large, but finite subsystems, each described by the Drude-Ullersma model. These subsystems are coupled to each other through a quantum mediator. As long as the subsystems are large enough, so that their energy changes slowly in comparison with the relaxation rate of the mediator's energy, Fourier's law follows as a differential form of the energy continuity equation. It is important to notice that at no point this derivation relies on any assumptions outside the framework of the Drude-Ullersma model. Thus, it may be considered as one of the few examples of rigorous derivation of Fourier's law from the first principles, at least on the time scale that leaves the modes of the thermal baths in thermal equilibrium.

We have applied our results to explain the observed anomalously large heat flux between STM tip and substrate. Our conclusion is that the effect is due to the mediating role of the $\mathrm{CO}$ molecule placed in the tip-substrate gap. We also outlined the approach by which our model can be applied in order to understand thermal conductivity of highly disordered substances - the minimum thermal conductivity - and to the non-equilibrium Josephson junction.

\section{ACKNOWLEDGMENTS}

The authors wish to acknowledge support from the Air Force Office of Scientific Research. One of the authors (G.Y.P.) is supported by the National Research Council Senior Associateship Award at the Air Force Research Laboratory.

\section{APPENDIX}

In the second way, at $t<0$, the dynamical variables $x_{2 i}(t)$ and $p_{2 i}(t)$ of the second bath are determined by relations to (32) and (33), while $x_{1 i}(t)$ and $x_{1 i}(t)$, which now incorporate the quantum system, are determined as

$x_{1 i}(t)=\sum_{k=0} \sqrt{\frac{\hbar}{2 m_{\nu i} \nu_{1 k}}} e_{i}^{k}\left(a_{1 k}^{+} e^{i \nu_{1 k} t}+a_{1 k} e^{-i \nu_{1 k} t}\right)$ 
and $p_{1 i}(t)=m_{1 i} \dot{x}_{1 i}(t)$. Here $e_{i}^{k}$ are orthonormal eigenvectors [7]:

$$
e_{i}^{k}=\sqrt{\frac{D^{2}+\nu_{k}^{2}}{D^{2}+\omega_{i}^{2}}} \frac{2 \Delta \omega_{i} \sin \phi\left(\omega_{k}\right)}{\pi\left(\omega_{i}^{2}-\nu_{k}^{2}\right)} .
$$

Taking into account (34) and A101, one finds

$$
\begin{aligned}
& \left\langle x_{1 i}(0) x_{1 j}(0)\right\rangle=\frac{\hbar}{2 \sqrt{m_{1 i} m_{1 j}}} \sum_{k} \frac{e_{i}^{k} e_{j}^{k}}{\nu_{k}} \operatorname{coth} \frac{\beta_{1} \hbar \nu_{k}}{2},(\mathrm{~A} 103) \\
& \left\langle p_{1 i}(0) p_{1 j}(0)\right\rangle=\frac{\hbar \sqrt{m_{1 i} m_{1 j}}}{2} \sum_{k} \nu_{k} e_{i}^{k} e_{j}^{k} \operatorname{coth} \frac{\beta_{1} \hbar \nu_{k}}{2},(\mathrm{~A} 104)
\end{aligned}
$$

and $\left\langle p_{1 i}(0) x_{1 j}(0)+x_{1 j}(0) p_{1 i}(0)\right\rangle=0$ as before. Using these relations, the first two sums in (28) can be recast into

$$
\begin{array}{r}
\frac{1}{2 m} \sum_{i=1} \frac{C_{1 i}}{m_{1 i}} \cos \left(\omega_{i} t\right) j_{1}^{(a)}=\frac{4 \hbar \gamma_{1} D^{2}}{\pi^{3} m} \sum_{k} \Delta \nu_{k}\left(D^{2}+\nu_{k}^{2}\right) \\
\quad \times \sin ^{2} \phi_{k} \operatorname{coth} \frac{\beta_{1} \hbar \nu_{k}}{2} \mathcal{F}_{1}^{(a)}\left(\nu_{k}\right) \mathcal{F}_{2}^{(a)}\left(\nu_{k}\right)
\end{array}
$$

and

$$
\begin{aligned}
\frac{1}{2 m} \sum_{i=1} & C_{1 i} \omega_{i} \sin \left(\omega_{i} t\right) j_{1}^{(b)}=\frac{4 \hbar \gamma_{1} D^{2}}{\pi^{3} m} \sum_{k} \Delta \frac{D^{2}+\nu_{k}^{2}}{\nu_{k}} \\
& \times \sin ^{2} \phi_{k} \operatorname{coth} \frac{\beta_{1} \hbar \nu_{k}}{2} \mathcal{F}_{1}^{(b)}\left(\nu_{k}\right) \mathcal{F}_{2}^{(b)}\left(\nu_{k}\right) .
\end{aligned}
$$

In $\mathrm{A} 105$ and $\mathrm{A} 106$,

$$
\begin{gathered}
\mathcal{F}_{1}^{(a)}=\sum_{i=1} \frac{\Delta \omega_{i}^{2} \cos \omega_{i} t}{\left(D^{2}+\omega_{i}^{2}\right)\left(\omega_{i}^{2}-\nu^{2}\right)} \\
\mathcal{F}_{2}^{(a)}=\sum_{n=1,2,3 ; i=1} \frac{g_{n} \Delta \omega_{i}\left[\mu_{n} \sin \omega_{i} t-\omega_{i} \cos \omega_{i} t\right]}{\left(\mu_{n}^{2}+\omega_{i}^{2}\right)\left(D^{2}+\omega_{i}^{2}\right)\left(\omega_{i}^{2}-\nu^{2}\right)}
\end{gathered}
$$

$$
\mathcal{F}_{1}^{(b)}=-\partial_{t} \mathcal{F}_{1}^{(a)}, \quad \mathcal{F}_{2}^{(b)}=-\partial_{t} \mathcal{F}_{2}^{(a)}
$$

Using [57], the above summations can be carried out accurately and the result is

$$
\begin{gathered}
\mathcal{F}_{1}^{(a)}(\nu)=\frac{\pi}{2} \frac{\nu}{D^{2}+\nu^{2}} \frac{\cos (\nu t+\phi(\nu))}{\sin \phi(\nu)}, \\
\mathcal{F}_{2}^{(a)}(\nu)=\frac{\pi}{2} \frac{1}{D^{2}+\nu^{2}} \sum_{n=1}^{3} \frac{f_{n}}{\mu^{2}+\nu^{2}} \times \\
{\left[\mu_{n} \frac{\sin (\nu t+\phi(\nu))}{\sin \phi(\nu)}-\nu \frac{\cos (\nu t+\phi(\nu))}{\sin \phi(\nu)}\right],}
\end{gathered}
$$

and $\mathcal{F}_{1}^{(b)}(\nu), \mathcal{F}_{2}^{(b)}(\nu)$ are determined from A109). In the derived expressions we disregarded all contributions that are exponentially decaying in time. As in the first way, after substituting $\mathcal{F}_{1,2}^{(a, b)}$ into $\mathrm{A} 105$ and $\mathrm{A} 106$ contributions that contain the product $\sin (\nu t+\phi(\nu)) \cos (\nu t+\phi(\nu))$ cancel each other, and the other time-dependent terms will be proportional to $\sin ^{2}(\nu t+\phi(\nu))+\cos ^{2}(\nu t+\phi(\nu))=1$. As is also clear, the coefficient in the product $\mathcal{F}_{1}^{(a, b)} \mathcal{F}_{2}^{(a, b)}$ is inverse proportional to $\sin ^{2} \phi_{k}$ and is canceled by similar factors in A105 and A106. This eliminates the dependence on initial conditions related to whether the central particle was initially connected or not to the first bath. These observations prove explicitly the existence of the steady-state in the presented mode and its uniqueness. Finally, replacing the summation over $k$ by the integral results in the same expression (40) for $\left\langle\mathcal{P}_{\nu}\right\rangle^{(a b)}$.
[1] A. Dhar, Advances in Physics 57, 457 (2008).

[2] Y. Dubi and M. Di Ventra, Review of Modern Physics 83, 131 (2011).

[3] Molecular Electronics, edited by J. Jortner and M. Ratner (Blackwell Science, Oxford, 1997).

[4] P. Hanggi, M. Ratner, and S. Yalikari, Chem. Phys. 281, 111 (2002).

[5] A.O. Caldeira and A.J. Leggett, Physica 121 A, 587 (1983).

[6] A.E. Allahverdyan and Th. M. Nieuwenhuizen, Phys. Rev. Lett. 85, 1799 (2000).

[7] Th. M. Nieuwenhuizen and A. E. Allahverdyan, Phys. Rev. E 66, 036102 (2002).

[8] M. Michel, G. Mahler, and J. Gemmer, Phys. Rev. Lett. 95, 180602 (2005).

[9] Y. Dubi and M. Di Ventra, Phys. Rev. E 79, 042101 (2009).

[10] M. Michel, M. Hartmann, J. Gemmer, and G. Mahler, Eur.
Phys. J. B 34, 325 (2003).

[11] D. Segal, A. Nitzan, and P. Hanggi, J. Chem. Phys 119, 6840 (2003).

[12] M. Michel, J. Gemmer, and G. Mahler, Int. J. Mod. Phys. B 20, 4855 (2006). and references therein.

[13] K. Saito, Europhys. Lett. 61, 34 (2003).

[14] F. Bonetto, J. L. Lebowitz, and L. Rey-Bellet, Fourier law: A challenge to theorists, in Mathematical Physics 2000, edited by A. Focas, A. Grigoryan, T. Kibble, and B. Zagarlinski (Imperial College Press, London, 2000).

[15] I.R. Senitzky, Phys. Rev. 119, 670 (1960).

[16] H. Mori, Prog. Theor. Phys. 33, 423 (1965).

[17] G.W. Ford, M. Cac, and P. Mazur, J. Math. Phys. 6, 504 (1965).

[18] H. Haken, Rev. Mod. Phys. 47, 67 (1975).

[19] Y.L. Klimontovich, Statistical Theory of Open Systems (Kluwer, Amsterdam, 1997). 
[20] U. Zürcher and P. Talkner, Phys. Rev. A 42, 3278 (1990).

[21] K. Saito, S. Takesue, and S. Miyashita, Phys. Rev. E 61, 2397 (2000).

[22] A. Dhar and B.S. Shastry, Phys. Rev. B 67, 195405 (2003).

[23] T. Prosen, New Journal of Physics 10, 043026 (2008).

[24] T. Prosen and B. Žunkovič, New Journal of Physics 12, 025016 (2010).

[25] I. Altfeder, A.A. Voevodin, and A.K. Roy, Phys. Rev. Lett. 105, 166101 (2010).

[26] G.W. Ford, J.T. Lewis, and R.F. O'Connell, Phys. Rev. A 37 4419 (1988).

[27] P. Ullersma, Physica (Utrecht) 32, 27 (1966); 32, 56 (1966); 32, 74 (1966); 32, 90 (1966).

[28] U. Weiss, Quantum Dissipative Systems ( World Scientific, Singapore, 1993).

[29] R.V. Churchill, Operational mathematics, 2nd ed. (McGrawHill, New York, 1972).

[30] A. Casher and J.L. Lebowitz, J. Math. Phys. 12, 1701 (1971).

[31] R.J. Rubin and W.L. Greer, J. Math. Phys. 12, 1686 (1971).

[32] R. Benguria and M. Kac, Phys. Rev. Lett. 46, 1 (1981).

[33] A. Dhar and K. Wagh, Eur. Phys. Lett. 79, 60003 (2007).

[34] A. Dhar and D. Sen, Phys. Rev. B 73, 085119 (2006).

[35] H. Dammak, Y. Chalopin, M. Laroche, M. Hayoun, and J.-J. Greffet, Phys. Rev. Lett. 103, 190601 (2009).

[36] L.D. Landau and E.M. Lifshitz, Statistical Physics, Part 1 (Pergamon Press, London, 1980).

[37] E. M. Lifshitz and L. P. Pitaevskii, Physical Kinetics (Pergamon Press, London, 2002).

[38] M. Bolsterli, M. Rich, and W. M. Visscher, Phys. Rev. A 1, 1086 (1970).
[39] M. Rich, and W. M. Visscher, Phys. Rev. B 11, 2164 (1975).

[40] M. Bandyopadhyay and D. Segal, Phys. Rev. E 84, 011151 (2011).

[41] We thank I. Altfeder for clarifying to us some of the details of the experiment.

[42] Hirschfelder, Curtiss and Bird, Molecular Theory of Gases and Liquids (Wiley, New York, 1954).

[43] G. Bylholder and R. Sheets, J. Phys. Chem. 74, 4335 (1970).

[44] J. P. Wolfe, Imaging Phonons:Acoustic Wave Propagation in Solids (Cambridge University Press, New York, 1998).

[45] D.G. Cahill, S.K. Watson, and R.O. Pohl, Phys. Rev. B 46, 6131 (1992).

[46] P. B. Allen and J. L. Feldman, Phys. Rev. B 48, 12581 (1993).

[47] W.P. Hsieh, M.D. Losego, P.V. Braun, S. Shenogin, P. Keblinski, and D.G. Cahill, Phys. Rev. B 83, 174205 (2011).

[48] A.F. Ioffe and A.R. Regel, Prog. Semicond. 4, 237 (1960).

[49] N.F. Mott, Philos. Mag. 26, 1015 (1972).

[50] D.J. Thouless, Phys. Rep. 13C, 93 (1974).

[51] E. Abrahams, P.W. Anderson, D. C. Licciardello, and T.V. Ramakrishnan, Phys. Rev. Lett. 42, 673 (1979).

[52] P. A. Lee and T.V. Ramakrishnan, Rev. Mod. Phys. 57, 287 (1985).

[53] G. A. Levin, Phys. Rev. B 70, 064515 (2004).

[54] R.C. Zeller and R.O. Pohl, Phys. Rev. B 4, 2029 (1970).

[55] K.K. Likharev, Sov. Phys. Usp. 26(1), 87 (1983).

[56] K.K. Likharev, Rev. Mod. Phys. 51(1), 101 (1979).

[57] A.P. Prudnikov, Y.A. Brychkov, and O.I. Marichev, Integrals and Series, Vol. 1: Elementary Functions (Gordon and Breach, New York, 1986). 